EPJ manuscript No.

(will be inserted by the editor)

\title{
Towards High-Energy Neutrino Astronomy
}

\section{A Historical Review}

\author{
Christian Spiering ${ }^{a}$ \\ DESY, Platanenallee, D-15738 Zeuthen
}

\begin{abstract}
The search for the sources of cosmic rays is a three-fold assault, using charged cosmic rays, gamma rays and neutrinos. The first conceptual ideas to detect high energy neutrinos date back to the late fifties. The long evolution towards detectors with a realistic discovery potential started in the seventies and eighties, with the pioneering works in the Pacific Ocean close to Hawaii and in Lake Baikal in Siberia. But only now, half a century after the first concepts, such a detector is in operation: IceCube at the South Pole. We do not yet know whether with IceCube we will indeed detect extraterrestrial high energy neutrinos or whether this will remain the privilege of next generation telescopes. But whatever the answer will be: the path to the present detectors was a remarkable journey. This review sketches its main milestones.
\end{abstract}

\section{Introduction}

The year 2012 marks the hundredth anniversary of the detection of cosmic rays by Viktor Hess [Hess 1912. As we know today, cosmic rays consist of protons and nuclei of heavier elements; electrons contribute only on the percent level. Since cosmic rays are electrically charged, they are deflected by cosmic magnetic fields on their way to Earth. Precise pointing - i.e. astronomy - is only possible with electrically neutral, stable particles: electromagnetic waves (i.e. gamma rays at the energies under consideration) and neutrinos. High energy neutrinos, with energies much beyond a GeV, must be emitted as a by-product of collisions of charged cosmic rays with matter. Actually, only neutrinos provide incontrovertible evidence for acceleration of hadrons since gamma rays may also evolve from inverse Compton scattering of accelerated electrons and other electromagnetic processes.

Since neutrinos can escape much denser celestial environments than light, they can be tracers of processes which stay hidden to traditional and gamma ray astronomy. At the same time, however, their extremely low reaction cross section makes their detection a challenge $\left(\sigma_{\nu p} \sim E_{\nu} \times 10^{-38} \mathrm{~cm}^{2}\right.$, with $E_{\nu}$ in $\left.\mathrm{GeV}\right)$.

Neutrino astronomy is reality already now in the low-energy sector, where the detection of neutrinos from the Sun and the Supernova SN 1987A has been accomplished and was honored by the 2002 Nobel Prize for physics. Figure 1 shows a compilation of the spectra of dominant natural and artificial neutrino fluxes.

\footnotetext{
${ }^{a}$ e-mail: christian.spiering@desy.de
} 


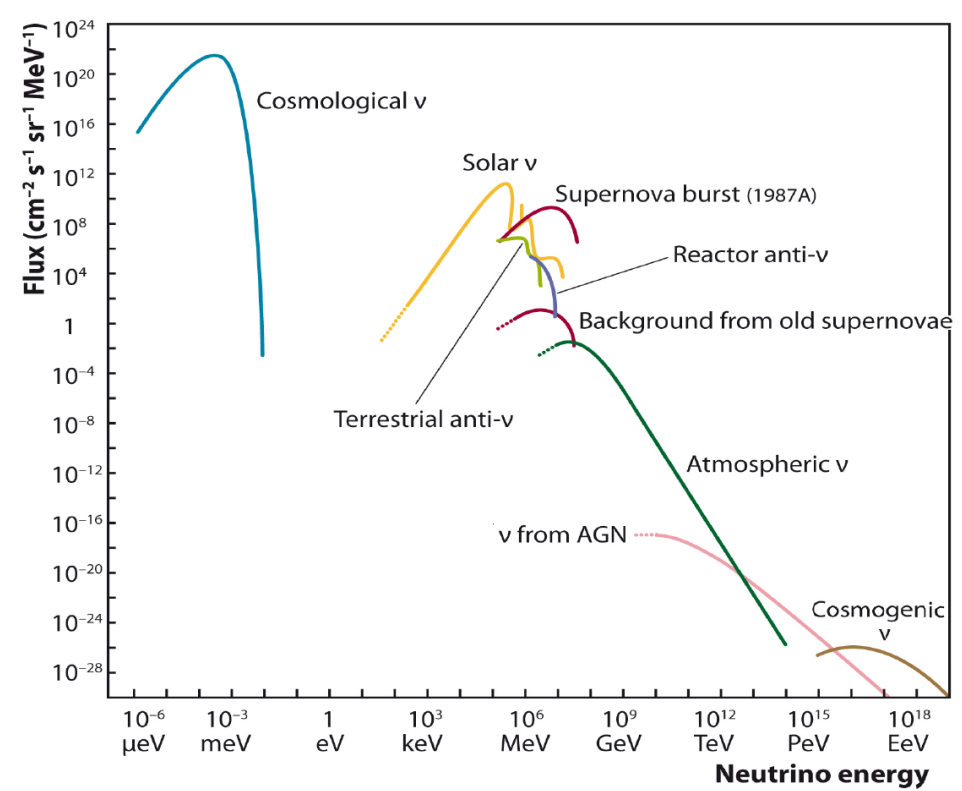

Fig. 1. Measured and expected fluxes of natural and reactor neutrinos (see text for explanations). The energy range from $\mathrm{keV}$ to several $\mathrm{GeV}$ is the domain of underground detectors. The region from tens of $\mathrm{GeV}$ to about $100 \mathrm{PeV}$, with its much smaller fluxes, is addressed by Cherenkov light detectors underwater and in ice. The highest energies are only accessible with huge detector volumes and methods described in section 12 .

The range of $\mu \mathrm{eV}$ and $\mathrm{meV}$ is that of cosmological (or "relic") neutrinos, i.e. the 1.9 Kelvin neutrino counterpart to the 2.7 Kelvin cosmic microwave background. No practicable idea exists on how to detect these neutrinos, since their reaction cross section as well as the energy of the recoil products from their interactions are frustratingly small.

The keV-MeV range is populated by neutrinos from the Sun, from supernovae, from nuclear reactors and the from the interior of the Earth. Neutrinos from a nuclear reactor first recorded in 1956 by Clyde Cowan and Frederick Reines Cowan 1956 mark the discovery of neutrinos. It was acknowledged with the 1995 Nobel Prize for physics. Solar neutrinos have been measured first by Ray Davis in 1968 Davis 1968 in the Homestake mine in USA. The apparent deficit of neutrinos observed by Davis the long-standing "solar neutrino puzzle" - could eventually be explained by neutrino oscillations which transform a large part of the original solar electron neutrinos to muon and tau neutrinos; with respect to these, the detector of Davis and many of its successors were blind. Supernova neutrinos from the supernova 1987A in the Large Magellanic Cloud have been recorded at February 23, 1987 by three detectors: Kamiokande in Japan, IMB in the USA (both water Cherenkov detectors) and the Baksan scintillation detector in Russia. The Nobel Prize for physics 2002 was awarded to Masatoshi Koshiba (spokesman of the Kamioka collaboration) and Ray Davis, for "pioneering contributions to astrophysics, in particular the detection of neutrinos from the Sun and a supernova". Neutrinos from radioactive decay processes in the interior of the Earth ("geo-" or "terrestrial" neutrinos) have been identified only recently Araki 2005 Bellini 2010. 
Next on the energy scale come "atmospheric neutrinos" created in cosmic ray interactions in the Earth's atmosphere. They have been detected in 1965 and will be in the focus of section 2 ,

The highest energies are the domain of neutrinos from sources like supernova remnants, Gamma Ray Bursts or Active Galactic Nuclei (marked AGN in the figure) or from interactions of ultra-energetic protons with the $2.7 \mathrm{~K}$ cosmic microwave background (marked "cosmogenic") Berezinsky \& Zatsepin 1970. These cosmic neutrinos will hopefully be detected by neutrino telescopes in this decade, even though predictions for their fluxes are uncertain by orders of magnitude in many cases.

This review is about neutrinos related to cosmic rays. First ideas to detect extraterrestrial high energy neutrinos data back to the end of the fifties, i.e. we look back to a journey of more than fifty years. I will focus to the first four decades and keep the developments of the last decade comparatively short. I refer the reader to the 2011 review of the field Katz \& Spiering 2011] for more detailed information on actual results and plans for future detectors.

\section{From first concepts to the detection of atmospheric neutrinos}

The initial idea of neutrino astronomy beyond the solar system rested on two arguments: The first was the expectation that a supernova stellar collapse in our galaxy would be accompanied by an enormous burst of neutrinos in the $5-10 \mathrm{MeV}$ range. The second was the expectation that fast rotating pulsars must accelerate charged particles in their Tera-Gauss magnetic fields. Either in the source or on their way to Earth they must hit matter, generate pions and neutrinos as decay products of the pions.

$$
p+\text { nucleus } \rightarrow \pi+X \quad \text { and } \quad \pi \rightarrow \mu+\nu
$$

The first ideas to detect cosmic high energy neutrinos underground or underwater date back to the late fifties. In the 1960 Annual Review of Nuclear Science, Kenneth Greisen and Frederick Reines discussed the motivations and prospects for such detectors. In his paper entitled Cosmic Ray Showers [Greisen 1960, Greisen writes:

Let us now consider the feasibility of detecting the neutrino flux. As a detector, we propose a large Cherenkov counter, about $15 \mathrm{~m}$ in diameter, located in a mine far underground. The counter should be surrounded with photomultipliers to detect the events, and enclosed in a shell of scintillating material to distinguish neutrino events from those caused by $\mu$ mesons. Such a detector would be rather expensive, but not as much as modern accelerators and large radio telescopes. The mass of the sensitive detector could be about 3000 tons of inexpensive liquid.

Later he estimates the rate of neutrino events from the Crab Nebula as one count per three years and optimistically concludes:

Fanciful though this proposal seems, we suspect that within the next decade cosmic ray neutrino detection will become one of the tools of both physics and astronomy.

F. Reines in his article Neutrino Interactions Reines 1960 is more conservative with respect to extraterrestrial neutrinos:

At present no acceptable theory of the origin and extraterrestrial diffusion exists so that the cosmic neutrino flux can not be usefully predicted. 
At this time, he could not be aware of the physics potential of atmospheric neutrinos and continues:

The situation is somewhat simpler in the case of cosmic-ray neutrinos ("atmospheric neutrinos" in present language. C.S.) - they are both more predictable and of less intrinsic interest.

In the same year, on the 1960 Rochester Conference, Moisei Markov published his groundbreaking idea Markov 1960.

...to install detectors deep in a lake or a sea and to determine the direction of charged particles with the help of Cherenkov radiation.

This appeared to be the only way to reach detector volumes beyond the scale of $10^{4}$ tons.

During the sixties, no predictions or serious estimates for neutrino fluxes from cosmic accelerators were published. Actually, many of the objects nowadays considered as top candidates for neutrino emission were discovered only in the sixties and seventies (the first quasar 1963, pulsars 1967, X-ray binaries with a black hole 1972, gamma ray bursts 1973). The situation changed dramatically in the seventies, when these objects were identified as possible neutrino emitters, triggering an enormous amount of theoretical activity.

Different to extraterrestrial neutrino fluxes, the calculation of the flux of atmospheric neutrinos became more reliable. The idea that neutrinos might be generated in air showers from cosmic rays, astonishingly, dates back to 1936. This was a only two years after Enrico Fermi had published his first paper on the theory of $\beta$ decay Fermi 1934 and six years after Wolfgang Pauli had postulated the neutrino Pauli 1930. In Heisenberg 1936 Werner Heisenberg writes:

Instead (of protons and neutrons, C.S.) Pauli's hypothetical 'neutrinos' should contribute substantially to the penetrating radiation. This is because in each shower ... neutrinos should be generated which then would lead to the generation of small secondary showers. The cross section for the generation of these secondary showers would likely not be much smaller than $10^{-26} \mathrm{~cm}^{2}$. Contrary to the low-energy neutrinos from $\beta$ decay one should be able to detect the energetic neutrinos from cosmic rays via their interactions.

Heisenberg's idea that neutrinos must be generated in showers was correct, but he overestimated the cross section by about ten orders of magnitude and therefore came to the wrong conclusion that the neutrinos would generate secondary showers and contribute to the multiplication process. The final prediction that neutrinos generated in atmospheric showers would be detectable is certainly true, but in another way than Heisenberg assumed: namely, by consequently filtering away all other particles from the showers, i.e. by going deep underground.

First serious estimates for the fluxes of atmospheric neutrinos were published in the early 1960s Zatsepin\&Kuzmin 1961 Cowsik 1963 Osborne 1965 Volkova\&Zatsepin 1965. Actually first calculations had been made in 1958 by Igor Zheleznykh, at that time a diploma student of Markov. In his diploma work he estimated the flux of atmospheric neutrinos and the flux of neutrinos from the Crab Nebula. The pioneering attempts of the two Moscow groups are described in detail in the recollections of Zheleznykh Zheleznykh 2006. His diploma work laid the basis of his common paper with Markov Markov \& Zheleznykh 1961. (Actually this paper had appeared already in 1960 in a collection of the Joint Institute of Nuclear Research, Dubna [Markov 1960a]). It is interesting to read in this paper a footnote acknowledging an communication from Georgi Zatsepin who was working on the same subject: 
We have been kindly informed by Professor G. Zatsepin that the contribution of neutrinos originating in $\mu$-decay may be essential for neutrinos of the order of several $\mathrm{GeV}$.

which refers to the decays

$$
\mu^{+} \rightarrow e^{+}+\bar{\nu}_{\mu}+\nu_{e} \quad \text { and } \quad \mu^{-} \rightarrow e^{-}+\nu_{\mu}+\bar{\nu}_{e}
$$

Actually this decay doubles the number of muon neutrinos and adds electron neutrinos. Only at higher energies muons have no time to decay before reaching the Earth where they are slowed down and stopped. In this case energetic neutrinos from muon decay can indeed be neglected. Reading the equation today one must keep in mind that in 1961, although the formula already appears in the papers of Markov and Zhelesnykh and of Zatsepin and Kuzmin, the existence of separate muon and electron neutrinos was not yet proven. The existence of the muon neutrino was confirmed only in 1962.

The real explosion of papers on atmospheric neutrinos, however, happened between 1980 and 1990 when the large underground detectors became operational and the field turned into a precision science (see section 6.2). Still, from the perspective of the sixties and early seventies, the study of atmospheric neutrinos appeared equally interesting as the search for extraterrestrial neutrinos Markov \& Zheleznykh 1961. Neutrino oscillations did not yet play a role in the discussions of the late sixties and appeared only in the seventies on the shopping list. However, atmospheric neutrinos offered a possibility to study neutrino cross sections in an energy region which was not accessible to accelerator experiments at that time. Using the language of the seventies, these studies would have given information on the mass of the intermediate $\mathrm{W}$-boson. Without proper guidance on the W-mass, these effects were expected to be visible already in the few-GeV range, and actually this was one of the main motivations to build the first underground neutrino detectors Zheleznykh 2006]. More generally, the availability of neutrinos with energies beyond what could realistically be expected from accelerator beams was recognized as a tempting method to search for phenomena beyond the standard model; however not by everybody! F. Reines notes in his summary of the Neutrino-81 conference Reines 1981:

Estimates of the atmospheric flux suggest that interactions of this source of $\geq 1 \mathrm{TeV}$ neutrinos might be usefully observed, although our accelerator-based colleagues are not keen on this as a source of new information.

Actually the attitude of the broader particle physics community with respect to the physics potential of atmospheric neutrinos changed only together with the detection of neutrino oscillations in the nineties.

Before studying atmospheric neutrinos they had to be detected. This was achieved in 1965, almost simultaneously, by two groups. One was led by Frederick Reines (CaseWitwatersrand group, later Case-Witwatersrand-Irvine, CWI). Three years earlier, Reines had asked Markov in Geneva, whether mines, tunnels or caverns existed in the Soviet Union which were suitable for underground experiments Markov 1993. Markov then charged his colleague A. Pomanski to investigate this case, with the result, that the were no such locations found. In the seventies Russia solved the problem in a unique brute-force attempt, by digging a special tunnel in the Baksan valley in the Caucasus.

The Reines group operated two walls of segmented liquid scintillator in the East Rand gold mine in South Africa, at a depth of $8800 \mathrm{~m}$ water equivalent. The configuration was chosen to identify horizontal muon tracks 1 . Between February and

\footnotetext{
1 The best signature to detect muon neutrinos is identifying upward moving muons, since they must be due to a particle being able to cross the Earth (see Fig 3). Since the Reines
} 
July 1965, seven such tracks were recorded, with a background of less than one event from muons not induced by neutrinos. It is interesting to note that the first of these tracks was recorded at February 23, 1965, exactly 22 years before the neutrinos from supernova SN1987A reached the Earth (23/2/1987). Some personal reminiscences of this experiment can be found in Kropp 1991.

The detector of the other group (a Bombay-Osaka-Durham collaboration) was operated in the Indian Kolar Gold Field (KGF) mine, at a depth of $7500 \mathrm{~m}$ water equivalent. It consisted of two walls of plastic scintillators and flash tubes. The KGF group started data taking nearly six months after the CW group, saw the first of three neutrino candidates two months later than Reines $(20 / 4 / 1965)$, but published two weeks earlier than the CW group: KGF at August 15, 1965 (submitted 12/7/1965 Achar 1965]), CW at August 30, 1965 (submitted 26/7/1965 [Reines 1965]). So, indeed Reines recorded the first cosmic neutrino ever, but the formal priority is with the KGF group; a historic race which had no losers but two winners.

With improved detectors, the two groups continued measurements for many years Crouch 1978 Krishnaswamy 1971, collecting a a total sample of nearly 150 neutrino events. The KGF group was the first to release a sky map (see Fig. 2). A comprehensive review of particle physics activities in the Kolar Gold Field mine, from the sixties to the nineties, is given in Narisimham 2004.

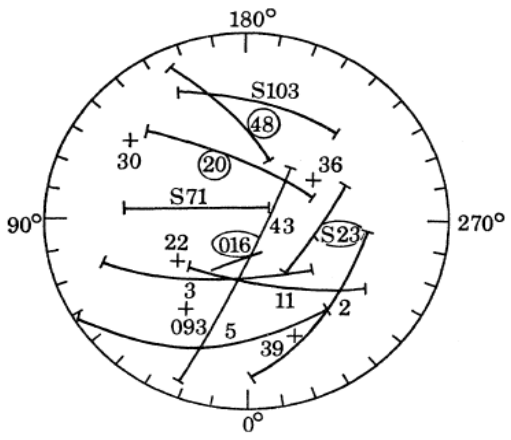

Fig. 2. The first neutrino sky map with the celestial coordinates of $18 \mathrm{KGF}$ neutrino events Krishnaswamy 1971. Due to uncertainties in the azimuth, the coordinates for some events are arcs rather than points. The labels reflect the numbers and registration mode of the events (e.g. "S" for spectrograph). Only for the ringed events the sense of the direction of the registered muon is known.

A third underground detector was operated from 1967 on by a group in Utah Bergeson 1967. It consisted of spark chambers and Cherenkov counters. The anomalies in muon depth-intensity which they reported in 1967-71 provided one of the initial motivations to build underwater detectors. In 1973, the Utah group reported also first results on neutrinos Bergeson 1973. Combining their energy spectra with those of the KGF group they concluded that the spectrum is consistent with a linear growth of the neutrino cross-section up to high energies and that the W-boson mass has to be large.

In 1978, the Baksan Neutrino Telescope (BNT) in the Caucasus started (partial) operation. It was followed by a phalanx of new detectors in the eighties, which mostly were motivated by the search for proton decay. The largest of them was the IMB detector which produced the first neutrino sky map of reasonable quality, with 187 events from 396 live-days Svoboda 1987.

The study of atmospheric neutrinos and of MeV-neutrinos from a galactic Supernova seemed to be feasible with detectors of a few hundred or thousand tons, with the

detector could measure the direction but not the sense of the direction, Reines had to focus to nearly horizontal tracks. At $8800 \mathrm{~m}$ depth water equivalent, the slant depth then is so large that it can be passed only by neutrinos, irrespective of the sense of the direction. 
main unknown being the rate at which those Supernovae occur. Predictions ranged from a several per millennium up to a few per century. Therefore a Supernova neutrino detector had better to be a multipurpose device with alternative goals, for instance atmospheric neutrinos or cosmic ray studies as reliable aims, combined with the highrisk aim to search for proton decay. Actually, the two water Cherenkov detectors which detected neutrinos from the supernova 1987A, IMB (USA) and Kamiokande (Japan), had both been funded for different primary purposes, most notably proton decay.

In contrast to investigating atmospheric and supernova neutrinos, the study of high-energy extraterrestrial neutrinos had the inherent risk that no reliable predictions for the expected fluxes could be made. Under these circumstances it appeared logical to tackle this problem with the largest devices conceivable, with underwater detectors of the kind which M. Markov had proposed in 1960.

Actually, a few proposals with elements of Markov's idea were made in the sixties. One of them in 1965 suggested to detect upward moving muons from neutrino interactions by observing a $10 \mathrm{~m}$ thick water target ("possibly in ocean or a lake") with downward looking photomultipliers Uberall \& Cowan 1965. Another one Bogatyrev 1971 ambitiously proposed three detectors, each with $10^{7}$ tons of distilled water a several $\mathrm{km}$ depth. The detectors should be widely spaced in order to allow for Supernova triangulation, i.e. determining the arrival direction of the neutrino swarm by measuring differences in arrival times at very distant locations ${ }^{2}$. Instead of housing photomultipliers in pressure resistant glass spheres of cm-thickness (the accepted method for all later deep underwater experiments), the author proposed to construct large pressure resistant phototubes and, moreover, to use wavelength shifters. With these two 1971 ideas he anticipated options which later were considered, although eventually abandoned, for the Baikal experiment, for DUMAND and for IceCube.

The first step from conceptual ideas to large-scale experimental efforts was done by the DUMAND project which will be described in the next section.

\section{DUMAND: from the first workshop to the DUMAND-II proposal}

\subsection{The early years}

The history of underwater neutrino telescopes starts with a project which eventually was cut off but left an incredibly rich legacy of ideas and technical principles: The DUMAND project. DUMAND stands for Deep Underwater Muon and Neutrino Detector. Its early history is excellently covered in a Personal history of the DUMAND project by Arthur Roberts Roberts 1992 .

At the 1973 International Cosmic Ray Conference (ICRC), a small group of physicists including F. Reines, J. Learned, H. Davis, P. Kotzer, M. Shapiro (all USA), G. Zatsepin (USSR) and S. Miyake (Japan) discussed a deep-water detector to clarify puzzles in muon depth-intensity curves. The anomalies reported by the group of $\mathrm{W}$. Keuffel in Utah ("Keuffel effect") Bergeson 1967 faded away, but it was obvious that such a detector could also work for neutrinos. An informal group of people to study such a detector was assembled, led by Reines, Roberts, Miyake and Learned.

The year 1975 saw the first of a - meanwhile legendary - series of DUMAND Workshops, this one at Washington State University Kotzer 1975. A survey of possible sites converged on the Pacific Ocean close to Hawaii, since it offered deep locations close to shore. A year later, a two-week workshop took place in Honolulu Roberts 1976]. At that time, three options for a deep sea array were discussed:

\footnotetext{
2 The principle of Supernova triangulation has been first proposed in a paper of Grigorij Domogatsky and Georgij Zatsepin Domogatsky \& Zatsepin 1965].
} 
- UNDINE (for "UNderwater Detection of Interstellar Neutrino Emission") was intended to detect neutrinos from supernova collapses from far beyond our own Galaxy (leaving the Galactic Supernovae to underground detectors) ${ }^{3}$. Based on overoptimistic assumptions of the neutrino energy spectrum, it was soon discarded.

- ATHENE (for "ATmospheric High-Energy Neutrino Experiment") was tailored to high-energy particle physics with atmospheric neutrinos.

- UNICORN (for "UNderwater Interstellar COsmic-Ray Neutrinos") had the primary goal to search for high-energy extraterrestrial neutrinos.

At the 1976 workshop and, finally, at the 1978 DUMAND workshop at the Scripps Institute in La Jolla Roberts 1978 the issue was settled in favor of an array which combined the last two options, ATHENE and UNICORN. It is noteworthy that concepts of UNDINE were revived in 1978 for IMB and later for Kamiokande, both in mines (see section 6.2).
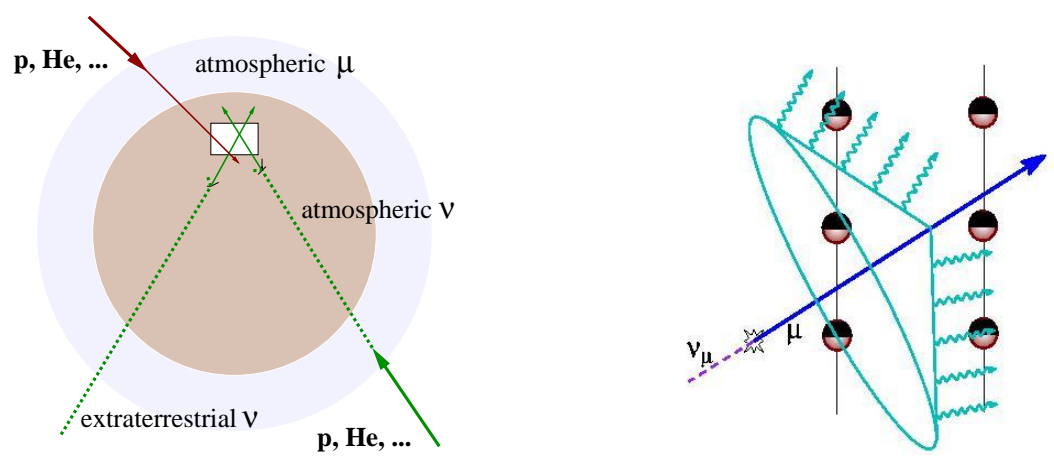

Fig. 3. Left: Sources of muons in deep underwater/ice detectors. Cosmic nuclei - protons (p), $\alpha$ particles (He), etc. - interact in the Earth atmosphere (light-colored). Sufficiently energetic muons produced in these interactions ("atmospheric muons") can reach the detector (white box) from above. Upward-going muons must have been produced in neutrino interactions. Right: Detection principle for muon tracks.

The principle of the detector was to record upward-traveling muons generated in charged current muon neutrino interactions. Neutral current interactions which produce no muons had been only discovered in 1973. They result in final-state charged particles of rather low energy and did not play a role for the design studies 4 . The upward signature guarantees the neutrino origin of the muon since no other particle can traverse the Earth. Since the sixties, a large depth was recognized as necessary in order to suppress downward-moving muons 5 which may be mis-reconstructed as upward-moving ones (Fig. 3. left). Apart from these, only one irreducible background to extra-terrestrial neutrinos remains: neutrinos generated by cosmic ray interactions in the Earth's atmosphere ("atmospheric neutrinos"). This background cannot be

\footnotetext{
3 Recently this idea was revived (without knowledge of the early DUMAND concept) with the proposal to drastically increase the photomultiplier density of a part of IceCube in order to monitor the full Virgo cluster and record several SN collapses per year.

${ }^{4}$ Muon-less events from charged current interactions of electron and tau-neutrinos were assumed to contribute only $1 / 3$ of the flux (oscillations leading to an 1:1:1 ratio have not yet been established at that time!) and much less with respect to the event rate. Moreover they have a less characteristic signature and were also not prioritized.

5 The ratio of downward to upward muons is of the order of $10^{6}$ at a depth of $1 \mathrm{~km}$.w.e. (km water equivalent) but only of the order $10^{4}$ at $4 \mathrm{~km} . \mathrm{w} . \mathrm{e}$.
} 
reduced by going deeper. On the other hand, it provides a standard calibration source and a reliable proof of principle.

The DUMAND design envisaged an array of photomultiplier tubes (PMTs) housed in transparent pressure spheres spread over a cubic kilometer (see Fig 4 left). The PMTs would record arrival time and amplitude of Cherenkov light emitted by muons or particle cascades. The spheres were to be attached to strings moored at the ground and held vertically by buoys. From the arrival times, the direction of the muon track can be reconstructed, and it turned out that a directional accuracy of 1 degree is achievable. This is of a similar size as the kinematic smearing between neutrino and muon direction and allows for neutrino tracing, i.e. for neutrino astronomy (see Fig. 3 , right). I refer to SSpiering 2011 for a detailed description of the functional principle, the technical realizations and the various event signatures in underwater/ice neutrino telescopes.

Naturally, the idea to construct a cubic-kilometer detector with more than 20000 large-size photomultipliers (see Fig. 4) challenged technical and financial possibilities. A. Roberts remembers Roberts 1992:

The 1978 DUMAND Standard Array, on closer examination, assumed more and more awesome proportions. ... 1261 sensor strings, each with 18 complex sensor modules ... to be deployed on the ocean bottom at a depth of $5 \mathrm{~km}$ ! The oceanographers were amazed - this project was larger than any other peacetime ocean project by a factor of the order of 100 . The size of the array was based on relatively scant information on the expected neutrino intensities and it was difficult to justify in detail; the general idea was that neutrino cross section are small and high-energy neutrinos are scarce, so the detector had better be large.

Confronted with the oceanographic and financial reality, the $1.26 \mathrm{~km}^{3}$ array was abandoned. A half-sized configuration (1980) met the same fate, as did a much smaller array with 756 phototubes (1982). The latter design was comparable in size to the AMANDA detector at the South Pole (see Sect.8) and the ANTARES telescope in the Mediterranean Sea, close to Toulon (see Sect. 9). What finally emerged as a technical project was a 216-phototube version, dubbed DUMAND-II or "The Octagon" (eight strings at the corners of an octagon and one in the center), $100 \mathrm{~m}$ in diameter and $230 \mathrm{~m}$ in height Bosetti 1988] (see Fig. 4). The plan was to deploy the detector $30 \mathrm{~km}$ off the coast of Big Island, Hawaii, at a depth of $4.8 \mathrm{~km}$.

\subsection{Flux predictions}

The evolution of the detector design, largely following financial and technological boundary conditions, was the one side of the story. What about the flux predictions?

At the 1978 workshop first investigations on neutron star binary systems as point sources of high energy neutrinos were presented, specifically Cygnus X-3 (D. Eichler/ D. Schramm and D. Helfand in Roberts 1978]). The connection to the indications for sources of $\mathrm{TeV}-\gamma$-ray (none of them significant at that time!) was discussed by T. Weekes. At the same time, the possibilities for diffuse source detection were disfavored (R. Silberberg, M. Shapiro, F. Stecker).

The gamma-neutrino connection was discussed further by Venjamin Berezinsky at the 1979 DUMAND Workshop in Khabarovsk and Lake Baikal (see Learned 1979). He emphasized the concept of "hidden" sources which are more effectively (or only) detectable by neutrinos rather than by $\gamma$ rays. Among other mechanisms, Berezinsky also investigated the production of neutrinos in the young, expanding shell of a supernova which is bombarded by protons accelerated inside the shell ("inner neutrino 


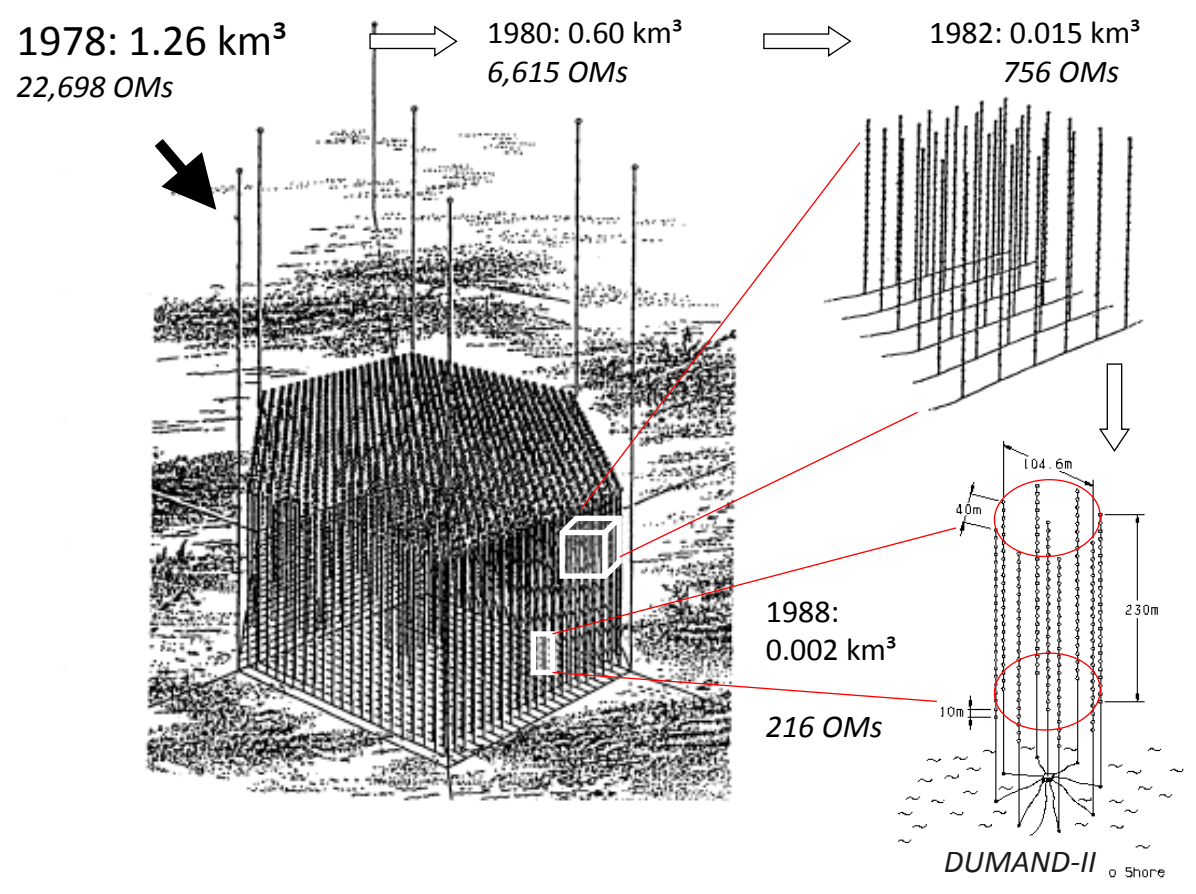

Fig. 4. The originally conceived DUMAND cubic kilometer detector and the phased downgrading to the 1988 plan for a first-generation underwater neutrino telescope DUMAND-II. See text for explanations.

radiation from a SN envelope") Berezinsky \& Priludsky 1977. He concluded that a $1000 \mathrm{~m}^{2}$ detector should be sufficient to detect high-energy neutrinos from a galactic supernova over several weeks or months after the collapse. Naturally, ten years later, in 1987, much attention was given to this model in the context of SN1987. But alas! - this supernova was at about $50 \mathrm{kpc}$ distance, more than five times further than the Galactic center. Moreover all underground detectors existing in 1987 had areas much smaller than $1000 \mathrm{~m}^{2}$. Therefore the chances to see inner neutrino radiation from the envelope were rather small, and actually "only" the MeV burst neutrinos and no high energy neutrinos have been recorded.

A large number of papers on expected neutrino fluxes was published during the eighties. The fluxes were found to depend strongly a) on the energy spectrum of the $\gamma$-ray sources which could only be guessed since the first uncontroversial $\mathrm{TeV}-\gamma$ observation was the Crab nebula in 1989 Weekes 1989, and b) on the supposed $\nu / \gamma$ ratio which depends on the unknown thickness of matter surrounding the source.

The uncertainty of expectations is reflected in Table 1 which is taken from the DUMAND-II proposal Bosetti 1988].

One sees that pessimistic and optimistic numbers differed by 2-3 orders of magnitude and left it open whether DUMAND-II would be able to detect neutrino sources or whether this would remain the realm of a future cubic kilometer array. Two years later, V. Berezinsky reiterated his earlier estimates that for neutrinos from a fresh neutron star a detector with an effective area of $1000 \mathrm{~m}^{2}$ (i.e. a large underground detector) would be sufficient, but that the detection of extragalactic sources would 


\begin{tabular}{|c|c|c|c|c|c|c|c|c|c|}
\hline \multirow{2}{*}{$\begin{array}{l}\text { Source } \\
\text { Name }\end{array}$} & \multirow{2}{*}{$\begin{array}{l}\text { RA } \\
(\mathrm{hh}: \\
\mathrm{mm}) \\
\end{array}$} & \multirow{2}{*}{$\begin{array}{r}\text { Dec } \\
(\mathrm{deg})\end{array}$} & \multirow{2}{*}{$\begin{array}{r}\text { Dist } \\
(\mathrm{kpc})\end{array}$} & \multirow{2}{*}{$\begin{array}{l}\gamma \text { En. } \\
(\mathrm{TeV})\end{array}$} & \multirow{2}{*}{$\begin{array}{r}\gamma \text { at Earth } \\
\left(\mathrm{cm}^{-2} \mathrm{~s}^{-1}\right)\end{array}$} & \multirow{2}{*}{$\begin{array}{l}\text { Luminos. } \\
\left(\operatorname{erg~s}^{-1}\right)\end{array}$} & \multirow{2}{*}{$\begin{array}{r}\text { Assumed } \\
\text { Spectr. } \\
\text { Index } \gamma \\
\end{array}$} & \multicolumn{2}{|c|}{$\mu / \mathrm{yr}$ in DUM-II } \\
\hline & & & & & & & & $\begin{array}{c}\varepsilon_{\nu / \gamma}=1 \\
\text { Min. } \gamma\end{array}$ & $\begin{array}{r}\varepsilon_{\nu / \gamma}=30 \\
\text { Max. } \gamma\end{array}$ \\
\hline Vela PSR & $08: 33$ & -45 & .5 & 5 & $1.8 \times 10^{-12}$ & $3 \times 10^{32}$ & $2.0-3.5$ & .1 & 1506 \\
\hline Vela X-1 & 09:00 & -40 & 1.4 & 1 & $2 \times 10^{-11}$ & $2 \times 10^{34}$ & $2.0-4.0$ & 2 & 126 \\
\hline Crab SNR & $05: 33$ & +22 & 2 & 2 & $1.1 \times 10^{-11}$ & $2 \times 10^{34}$ & $2.0-4.0$ & .2 & 438 \\
\hline Crab PSR & $05: 31$ & +21 & 2 & 1 & $7.9 \times 10^{-12}$ & $6 \times 10^{33}$ & $2.0-4.0$ & .06 & 38 \\
\hline Geminga & $06: 49$ & +18 & $.5-2.1$ & 6 & $9.5 \times 10^{-12}$ & $3 \times 10^{33}$ & $2.0-3.2$ & .49 & 1506 \\
\hline $4 \mathrm{U} 0115$ & $01: 15$ & +63 & 5 & 1 & $7.0 \times 10^{-11}$ & $6 \times 10^{35}$ & $2.0-4.0$ & .47 & 273 \\
\hline Her X-1 & $16: 57$ & +35 & 5 & 1 & $3 \times 10^{-11}$ & $3 \times 10^{35}$ & $2.0-4.0$ & .24 & 141 \\
\hline SS433 & 19:09 & +05 & 5 & 1 & $<10^{-10}$ & $<4 \times 10^{35}$ & $2.0-4.0$ & $<.88$ & $<510$ \\
\hline Cen X-3 & $11: 19$ & -60 & $5-10$ & 1 & $<5.2 \times 10^{-12}$ & $<2 \times 10^{34}$ & $2.0-4.0$ & $<.08$ & $<48$ \\
\hline Cyg X-3 & $20: 32$ & +41 & $\geq 11$ & 1 & $5.0 \times 10^{-11}$ & $3 \times 10^{36}$ & $2.1-4.0$ & .4 & 234 \\
\hline LMC X-4 & $05: 32$ & -66 & 55 & $10^{4}$ & $5 \times 10^{-15}$ & $1 \times 10^{38}$ & $2.0-4.0$ & 0.0001 & 0.048 \\
\hline M 31 & $00: 41$ & +41 & 670 & 1 & $2.2 \times 10^{-10}$ & $2 \times 10^{40}$ & $2.0-4.0$ & 1.8 & 1050 \\
\hline Cen $\mathrm{A}$ & $13: 24$ & -43 & 4400 & 3 & $4.4 \times 10^{-11}$ & $3 \times 10^{40}$ & $2.0-4.0$ & .14 & 6 \\
\hline $3 \mathrm{C} 273$ & $00: 12$ & +02 & $6 \times 10^{5}$ & 5 & $<9 \times 10^{-12}$ & $<3 \times 10^{45}$ & $2.0-3.3$ & $<.4$ & $<1506$ \\
\hline
\end{tabular}

Table 1. Tabulation of various $\gamma$-ray sources used for neutrino estimates (taken from Bosetti 1988). The last two columns give the number of upward muon neutrino events per year in DUMAND-II. The minimum numbers are obtained for a $\nu / \gamma$ ratio of 1 and flat gamma source spectra $(\operatorname{Min} \gamma)$, the maximum numbers for $\nu / \gamma=30$ and steep gamma spectra $(\operatorname{Max} \gamma)$. The spectral index $\gamma$ is the differential one.

require detectors of 0.1-1.0 $\mathrm{km}^{2}$ size Berezinsky 1990. DUMAND-II, with 25,000 $\mathrm{m}^{2}$ area, fell just below these values. Again citing A. Roberts Roberts 1992]:

These calculations serve to substantiate our own gut feelings. I have myself watched the progression of steadily decreasing size ... at first with pleasure (to see it become more practical), but later with increasing pain. ... The danger is, that if DUMAND II sees no neutrino sources, the funding agencies will decide it has failed and, instead of expanding it, will kill it.

In 1980, a feasibility study was started and supported by the University Hawaii. As a part of the study, a series of deep-ocean experiments was performed with the aim to test the detection concepts and measure the relevant water parameters. Actually, first site surveys had started already in 1978. For the first time, the attenuation length of sea water was measured with reasonable precision and the ambient light noise determined, including the study of stimulated bio-luminescence. Tests and investigations made during many cruises pushed ahead of oceanographic practice at that time. However, the ocean turned out to be a hostile place: in 1982 a test string was lost as its support cable broke in heavy seas, in 1984 another string failed to be released from its anchor at the bottom of the sea (but was recovered 18 months later).

After the initial series of site surveys and technical tests, in 1983 the Department of Energy (DOE) approved the funding for DOE-supported U.S. groups to deploy the "Short Prototype String" (SPS). With additional support from NSF, ICRR in Japan and the University of Bern in Switzerland, the SPS was conceived to develop and test the basic detector techniques, to further study the environmental effects, to demonstrate that muons can be reconstructed and to measure the muon vs. depth dependence. In 1987, this 7-phototube test string was successfully deployed for some hours from a a high-stability Navy vessel Babson 1990. It provided the measured muon intensity as a function of depth. Ironically, this most sustaining physics result from DUMAND returned, in a sense, to the initial idea of the 1973 ICRC.

After the successful SPS test, in 1988 the DUMAND-II proposal was submitted to DOE and NSF. The collaboration groups of this proposal were: UC Irvine, CalTech, U. Hawaii, Scripps Inst. Oceanology, U. Vanderbilt, U. Wisconsin (USA), U. Kinki, ICRR Tokyo (Japan), TH Aachen, U. Kiel (Germany) and U. Bern (Switzerland). DUMAND-II with its $100 \mathrm{~m}$ diameter and $230 \mathrm{~m}$ height, would have detected three down going muons per minute and about 3500 atmospheric neutrinos per year. 


\subsection{Technological solutions}

A wealth of technological solutions was found within the design study for the SPS, and many remained as a legacy for other neutrino telescopes. Some of the innovative solutions were only possible since basic technologies had only recently appeared on the market. Here I highlight two of them: a) the development of a highly sensitive photo-sensor and $b$ ) the development of fast data transmission using optical fibers.

The photomultiplier tube (PMT) had to be large (to collect much light), fast (to allow for fast timing and good muon angular resolution) and to have a good amplitude resolution (allowing identification of the 1-photoelectron (PE) peak and separation from noise and possibly from the 2-PE signals). 1-to-2 PE separation had the potential to separate hits due to light from ${ }^{40} \mathrm{~K}$ decays (nearly entirely 1-PE) from those from charged muons and is accomplished by PMTs with a high-gain first dynode.

A first approach to collect a maximum amount of light - long before the SPS phase - was the "Sea Urchin": a medium-sized PMT was foreseen to be centered in a commercially available 17 -inch glass pressure sphere filled with liquid. Spines made from radial glass tubes about $2 \mathrm{~cm}$ diameter and $3 \mathrm{~m}$ length and doped with wave length shifter would guide the light to the sphere - see Fig.5. However, such a design is highly fragile and turned out not to be feasible for underwater operation.

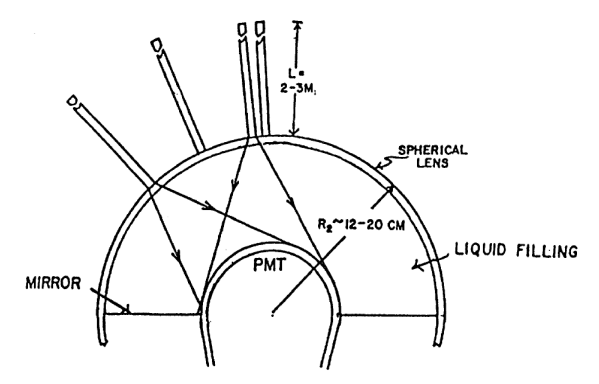

Fig. 5. The Sea-Urchin (Figure taken from [Roberts 1992]). See text for explanation.

Various innovative attempts were made but eventually discarded after Hamamatsu Comp. (Japan) committed to develop a spherical 15-inch PMT R2018, following a computer-simulated design developed by J. Learned. This PMT fitted into a 17-inch commercial pressure sphere which was tested down to $6700 \mathrm{~m}$ depth (Benthos company, USA). The PMT was embedded in silicone gel and shielded by a mu-metal grid against the Earth magnetic field. The only other practicable design for a light sensor was the PHILIPS "smart" photomultipler XP2600 van Aller 1986]. This PMT had a better amplitude resolution and was rather insensitive against the Earth magnetic field. I will sketch its operation principle in the context of the Baikal neutrino telescope, where a similar tube was developed.

Another example for applying brand-new techniques is the use of optical fibers for data transmission. The processed signal is sent as optical pulse through a multimode fiber cable. Fibers for use in undersea cables had been become available just in the late seventies. This was the second fortunate event with remarkable consequences since it removed the low-data-rate barrier imposed by shore cables with copper lines of $40 \mathrm{~km}$ length.

To feed the optical signal through the glass sphere, a special penetrator was designed by the collaboration since no connector matching the requirements was on the market (production by Diamond company, Switzerland). Remarkably, a decade 


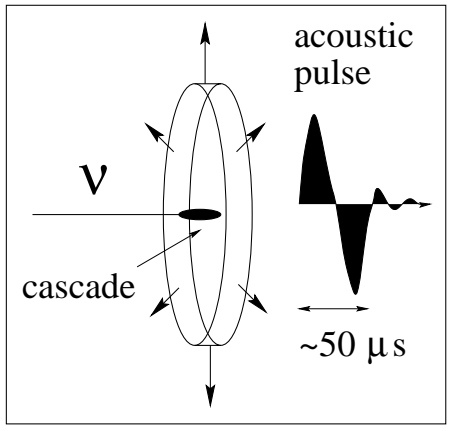

Fig. 6. Acoustic emission by a particle cascade.

later this connector had a revival, when the AMANDA experiment decided to replace the analog data transmission via electrical cables by optical fiber transmission (see below).

\subsection{Acoustic detection of neutrinos}

In 1957, the Soviet physicist Gurgen Askaryan had predicted the production of pressure waves by charged particles depositing energy in liquids or in solid media Askaryan 1957. Already at the second DUMAND Workshop DUMAND-1976 it was discussed whether this method could complement the detection of charged particles via their Cherenkov light. In the case of a particle cascade, its entire energy is deposited into the medium, mostly through ionization, and converted to heat on a time scale that is very short compared to the typical time scales relevant for generation and propagation of acoustic pulses. The effect is a fast expansion, generating a bipolar acoustic pulse with a width of a few ten microseconds in water or ice (see Fig. 6), corresponding to a peak signal power at $20 \mathrm{kHz}$. Transversely to the pencil-like cascade, the acoustic pulse propagates into the medium within a disk-shaped volume with a thickness corresponding to the cascade length of about $10 \mathrm{~m}$.

Since the attenuation length of sound in water is larger than that of light, the hope was that with comparably cheap hydrophones much larger volumes could be instrumented than with expensive photomultipliers. Therefore already in 1977, a special "Workshop on Acoustic Detection of Neutrinos" was organized in La Jolla Bradner 1977. Two years later, in 1979, the principle was experimentally proven with high-intensity proton beams at accelerators mimicking particle cascades [Learned 1979a]. However, it was rather quickly concluded that the acoustic method while possibly offering great volumes, had thresholds in the range of $100 \mathrm{PeV}$, higher than the only guaranteed source, namely atmospheric neutrinos. So no signal at all would be likely.

Since the late seventies, signal predictions have been considerably scaled down. Adding to this the meanwhile better understood backgrounds of the ambient and intrinsic noise, today the threshold for a reasonable spacing is estimated in the range of several $\mathrm{EeV}\left(10^{9} \mathrm{GeV}\right)$, see section 12 .

\section{The evolution of the Baikal project}

\subsection{The first years}

Russian participation in the DUMAND project was strong from the beginning and represented by names like A. Chudakov, V. Berezinsky, L. Bezrukov, B. Dolgoshein, 
A. Petrukhin and I. Zheleznykh. In 1979, the DUMAND Workshop was held in Khabarovsk and at Lake Baikal Learned 1979. However, in the context of the Soviet invasion in Afghanistan, in 1980 the Reagan administration terminated the cooperation. As A. Roberts remembers Roberts 1992]:

The severing of the Russian link was done with elegance and taste. We were told, confidentially, that while we were perfectly free to choose our collaborators as we liked, if perchance they included Russians it would be found that no funding was available.

Although A. Chudakov (together with F. Reines, O. Allkover, S. Myaki, J. Learned and M. Shapiro) still signed an open letter from July 26, 1980 which declares the formation of a DUMAND collaboration board Markov 1993, it was clear that Russia had to find its own way.

Also in 1980, Chudakov proposed to use the deep water of Lake Baikal in Siberia as the site for a "Russian DUMAND". The advantages of Lake Baikal seemed obvious: it is the deepest freshwater lake on Earth, with its largest depth at nearly 1700 meter, it is famous for its clean and transparent water, and in late Winter it is covered by a thick ice layer which allows installing winches and other heavy technique and deploying underwater equipment without any use of ships.

In 1981, first shallow-site experiment with small PMTs started. The chair of a dedicated laboratory at the Moscow Institute of Nuclear Research, Academy of Science of USSR (INR) became Grigorij Domogatsky, a theoretician, flanked by Leonid Bezrukov as leading experimentalist.

Soon a site in the Southern part of Lake Baikal was identified as suitable. It was about $30 \mathrm{~km}$ South-West from the outflow of Lake Baikal into the Angara river and approximately $60 \mathrm{~km}$ from the large city Irkutsk. A site at a distance of $3.6 \mathrm{~km}$ to shore and at a depth of about $1370 \mathrm{~m}$ was identified as the optimal location for a detector which would be installed at a depth of about 1.0-1.1 km. Detectors could be installed in a period between late February and early April from the ice cover, and operated over the full year via a cable to shore.

In the US, these efforts were noticed but obviously not understood as a competition to DUMAND. V.Stenger, who was the leading Monte-Carlo expert of the DUMAND project, repeatedly expressed his doubts that one could separate neutrinos from background in Lake Baikal. He argued that the lake was too shallow and the background of downward-going muons much too high. Therefore, the necessary cuts to reject the background would inevitably also strongly diminish the signal of upward going muons from neutrino interactions, with the exception of rather small and dense arrays.

After operation of first underwater modules with a 15-cm PMT in 1982, in the following year a small string was operated for several days. In 1984, a first stationary string was deployed (Girlanda-84) and recorded downward moving muons Bezrukov 1984. It consisted of three floors each with four PMTs in two pressuretolerant cylinders of glass-fiber enforced epoxy. At that time, no pressure tight glass spheres where available in the USSR. The end of the cylinders were closed by caps of plexiglass (see Fig.7). The PMT was a Russian tube (type FEU-49) with a $15 \mathrm{~cm}$ flat photocathode and modest amplitude and time resolution. An electrical cable connected the string to the shore.

The 1984 string was followed by another stationary string in 1986 (Girlanda-86). Data from this string were used to search for slowly moving bright particles like magnetic monopoles Domogatsky 1986. These particles had been introduced by P. Dirac in 1931 Dirac-1931. In the seventies they turned out to be a consequence of most variants of Grand Unified Theories (GUTs). A phase transition in the early Universe might have filled the Universe with a significant amount of monopoles. Typical 


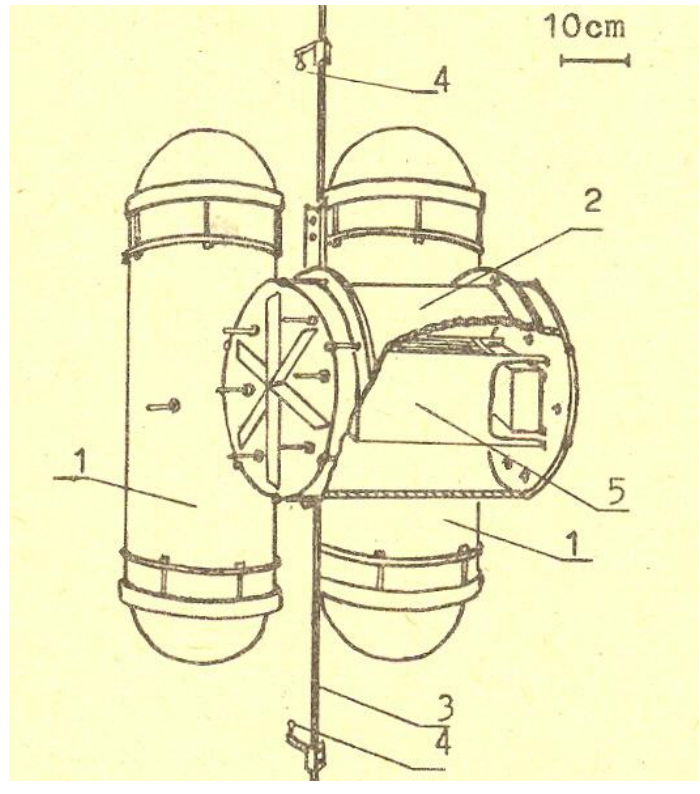

Fig. 7. A "svjaska" (Russian for "bundle") as used in GIRLANDA-86 for the monopole search, with 4 PMTs in 2 cylinders (1) covered by hemispheric plexiglass caps. The "backpack" module (2) contains electronics (5). Calibration LEDs (4) are fixed at the geophysical cable (3).

GUT versions predict monopoles with masses $10^{16} \mathrm{GeV}$ and more. These monopoles would have typical velocities of $v / c=10^{-4}-10^{-3}$. They might catalyze baryon decays along their path Rubakov 1981 which could be detected via the Cherenkov light from the decay particles. Other slowly moving exotic particles would be nuclearites (or "strangelets"), hypothetical aggregates of $u, d$ and $s$ quarks combined with electrons De Rujula \& Glashow 1984]. Nuclearites could have been produced in the primordial Universe or in certain astrophysical processes like the collision of neutron stars. They would induce a thermal shock wave along their path, the heated medium would emit Planck radiation and the effect would be a similar light pattern as for GUT monopoles. Data from Girlanda-86 set stringent limits on the flux of magnetic monopoles with high catalysis cross section Domogatsky 1986 and on the flux of nuclearites Belolaptikov 1998.

\subsection{Towards NT200}

Girlanda-84 took data for a total of 50 days and then sank down due to leaking buoys which held the string in vertical position. But also the cable penetrators through the epoxy cylinders as well as the cap-to-cylinder hermetic connection tended to leak and were a notorious source of headaches. Moreover, it was clear that the PMT used was much too small and too slow for a neutrino telescope. Therefore a technology using glass spheres and a new type of photo-sensor were developed.

The Russians started with testing the "smart" XP2600 from PHILIPS (see section 3 in Lake Baikal Bezrukov 1988. In parallel, the development of an equivalent Russian device, the QUASAR, was tackled, in cooperation with the EKRAN company in Novosibirsk. The QUASAR (Fig 8) is a hybrid device similar to the PHILIPS 2600 developed for the DUMAND project. Photoelectrons from a $370 \mathrm{~mm}$ diameter cathode $\left(\mathrm{K}_{2} \mathrm{CsSb}\right)$ are accelerated by $25 \mathrm{kV}$ to a fast, high-gain scintillator placed near 
the center of the glass bulb. The light from the scintillator is read out by a small conventional photomultiplier (type UGON). One photo-electron from the hemispherical photocathode yields typically 20 photoelectrons in the small photomultiplier. This high multiplication factor results in an excellent 1-PE resolution, clear distinction between 1-PE and 2-PE pulses, a time jitter as small as $2 \mathrm{~ns}$ and negligible sensitivity to the Earth's magnetic field Bagduev 1999.

In 1988, the Baikal experiment was approved as a long-term direction of research by the Soviet Academy of Sciences and the USSR government which included considerable funding. A full-scale detector (yet without clear definition of its size) was planned to be built in steps of intermediate detectors of growing size. In the same year 1988, the author's group from the East German Institute of High Energy Physics (IfH) in Zeuthen joined the Baikal experiment ${ }^{6}$.

After German unification in 1990, the Zeuthen group had access to the Western market and contributed with Jena glass spheres and some underwater connectors to the strings which were deployed in 1991 to 1993 (see below). In parallel, Russian spheres were developed in collaboration with industry, as well as penetrators and connectors which tolerated water depths down to $2 \mathrm{~km}$ - not suitable for large-depth Ocean experiments but sufficient for Lake Baikal.

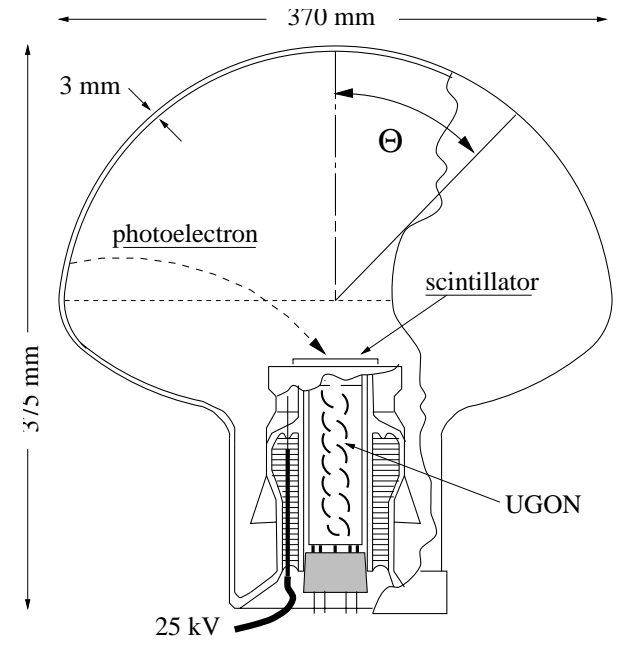

Fig. 8. The QUASAR-370 phototube

In 1989, a preliminary version of what later was called the NT200 project BAIKAL was developed, an array comprising approximately 200 optical modules. The final version of the project description was finished in 1992 [Sokalski \& Spiering 1992. At this time, the participating groups came from INR Moscow, Univ. Irkutsk, Moscow State Univ., Marine Techn. Univ. St. Petersburg, Polytechnical Institutes in Niszhni Novgorod and Tomsk, JINR Dubna, Kurchatov Inst.(Moscow), Limnological Inst. Irkutsk (all Russia), DESY-Zeuthen (Germany) and KFKI Budapest (Hungary).

NT200 (Fig. 9, left) is an array of 192 optical modules carried by eight strings which are attached to an umbrella-like frame consisting of $7 \mathrm{arms}$, each $21.5 \mathrm{~m}$ in length. The strings are anchored by weights at the lake floor and held in a vertical position by buoys at various depths. The configuration spans $72 \mathrm{~m}$ in height and $43 \mathrm{~m}$ in diameter. The finely balanced mechanics of this frame, with all its buoys, anchor

\footnotetext{
${ }^{6}$ As a result of the German unification, in 1992 IfH became part of the Deutsches Elektronensynchrotron, DESY.
} 
weights and pivoted arms is another stunning feature of the Baikal experiment. The detector is deployed (or hauled up for maintenance) within a period of about 6 weeks in February to April, when the lake is covered with a thick ice layer providing a stable working platform. It is connected to shore by several copper cables on the lake floor which allow for operation over the full year.

The optical modules with the QUASAR-370 phototubes are grouped pair-wise along a string. In order to suppress accidental hits from dark noise (about $30 \mathrm{kHz}$ ) and bio-luminescence (typically $50 \mathrm{kHz}$ but seasonally raising up to hundreds of $\mathrm{kHz}$ ), the two photo-multipliers of each pair are switched in coincidence. The time calibration is done using several nitrogen lasers in pressure-tight glass cylinders.

The construction of NT200 coincided with the decay of the USSR and an economically desperate period. Members of the collaboration and even some industrial suppliers had to be supported by grants from Germany; nevertheless many highly qualified experimentalists left the collaboration and tried to survive in the private sector. Over a period of three years, a large part of the food for the winter campaigns at Lake Baikal had to be bought in Germany and transported to Siberia. Still, a nucleus of dedicated Russian physicists heroically continued to work for the project. Under these circumstances, the construction of NT200 extended over more than five years. It started with the deployment of a 3-string array [Wischnewski 1993] with 36 optical modules in March/April 1993. The first two upward moving muons, i.e. neutrino candidates, were separated from the 1994 data. In 1996, a 96-OM array with four NT200 strings was operated Balkanov 1999] and provided the the first textbook neutrinos like the one shown in Fig 9 (right).
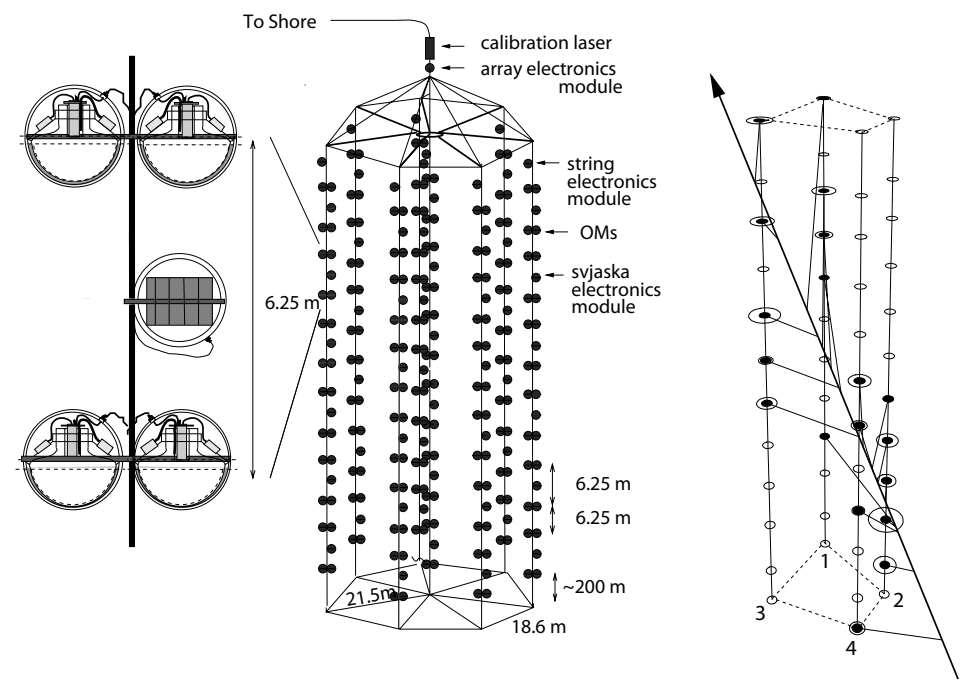

Fig. 9. Left: The Baikal Neutrino Telescope NT200. Right: One of the first upward moving muons from a neutrino interaction recorded with the 4-string stage of the detector in 1996 Balkanov 1999. The Cherenkov light from the muon is recorded by 19 channels.

NT200 was completed in April 1998 and has taken data since then. The basic components have been designed and built in Russia, notably the mechanics of the detector, the optical module, the underwater electronics and the cabling. The non-Russian contributions all came from DESY: the laser time calibration system, a 
transputer farm in the shore station for fast data processing, an online monitoring system and a special underwater trigger tailored to register slowly moving very bright particles (as GUT monopoles), not to mention the supply of Western electronics and glass spheres.

The small spacing of modules in NT200 leads to a comparably low energy threshold of about $15 \mathrm{GeV}$ for muon detection. About 400 upward muon events were collected over 5 years. This comparatively low number reflects the notoriously large number of failures of individual channels during a year rather than what would correspond to the effective area. Still, NT200 could compete with the much larger AMANDA for a while by searching for high energy cascades below NT200, surveying a volume about ten times as large as NT200 itself Aynutdinov 2006 Avronin 2009. In order to improve pattern recognition for these studies, NT200 was fenced in 2005-2006 by three sparsely instrumented outer strings ( 6 optical module pairs per string). This configuration is named NT200+ Aynutdinov 2006a, but suffered from several problems (from the new strings as well as from the meanwhile antiquated NT200 itself), so that no satisfying statistics and no convincing results have yet been obtained.

\section{The Venice Workshops and the 1992 Hawaii Workshop}

In 1988, Milla Baldo-Ceolin from University Padova organized the first "International Venice Workshop on Neutrino Telescopes", a conference series which developed to a discussion forum for new ideas and results in the field and a valuable archive of the stormy development of this branch of physics.

At the first meeting, R. March from Wisconsin explained a brand-new idea of F. Halzen (Wisconsin) and J. Learned (Hawaii) to detect neutrinos in ice (see section 6.3). Several talks presented new ideas on neutrino detectors at the surface which at that time seemed a promising possibility to avoid the difficulties of deep water detectors (see section 6.1). M. Koshiba (Tokyo University) made a strong case for imaging water detectors like Kamiokande and IMB, to which he attributed a firmer perspective than to the deep water projects like Baikal and DUMAND which used no imaging but timing technique. D. Samm from Aachen presented test results from the hybrid PHILIPS photomultiplier which at the same time already was being improved by Russian physicists (the QUASAR tube) and then constituted the basic element of the Baikal experiment (see sections 3 and 4 ).

Two years later, at the second Venice Workshop, results from data taken with the first of six modules the MACRO underground experiment in the Italian Gran Sasso Laboratory were reported. This experiment was going to be, together with Super-Kamiokande in Japan, the largest underground experiment (see section 6.2). J. Learned (Hawaii) and T. Gaisser (Delaware) discussed The Future of High Energy Neutrino Astronomy [Learned 1990] and Prospects for High Energy Neutrino Astronomy Gaisser 1990. While Moisei Markov and Frederick Reines, the visionary fathers of the field, had retired from active physics, Venyamin Berezinsky, Francis Halzen and John Learned became the driving and propagandistic motors of high-energy neutrino detection. Emerging from a round table discussion at the 1993 Venice Workshop, Halzen and Learned prepared a paper High Energy Neutrino Astronomy: Towards a $1 \mathrm{~km} 3$ Detector Halzen \& Learned 1993. In the summary they write:

Yet, we must acknowledge that those instruments now building for operation by 19957 will probably not be large enough to really undertake neutrino astronomy. It will require another step of about two orders of magnitude to

7 i.e. Baikal NT200, DUMAND-II and in addition (see below) AMANDA at the South Pole and NESTOR in the Mediterranean Sea 
be well into business, and for that it is not too early for dreams, plans, and studies.

Learned already in his 1990 talk in Venice Learned 1990 had pointed to the need for a cubic kilometer detector to do real astronomy but somewhat less reluctant supposed that

... in order to proceed with such grand visions we must have success in detecting the first astrophysical point sources of high energy neutrinos in the shorter term.

Over more than two decades, the Venice Workshops gathered hundreds of physicists to discuss neutrino physics, astrophysics and cosmology. The strong role of the workshop for the development of neutrino astronomy cannot be underestimated.

Another key meeting of the nineties was the Workshop on High-Energy Neutrino Astrophysics in Honolulu, Hawaii [Stenger \& Learned 1992]. It was attended by more than sixty experts in the field and was particularly important since it included a large number of astrophysicists which helped to get a better picture about the expected neutrino fluxes from astrophysical sources.

The workshop was focused to extragalactic rather than galactic sources. J. Learned and V.Stecker presented the number of muon and cascade events per year to be expected in DUMAND-II from unresolved and resolved Active Galactic Nuclei, see Table 2, The estimates from different models on unresolved AGN seem to differ by less than an order of magnitude and suggest a certain reliability of the predictions. This appears misleading since some models consider neutrino production in the core of AGN, some in the jets. Some models assume pion production in proton-proton collisions, others in proton collisions with ambient photon fields ( $\mathrm{p} \gamma$ collisions), some include both collision types. By today, all of these models have been excluded.

\begin{tabular}{|c|c|c|c|c|c|}
\hline Source & $\begin{array}{l}E_{\mu}> \\
100 \mathrm{GeV}\end{array}$ & $\begin{array}{l}E_{\mu}> \\
10 \mathrm{TeV}\end{array}$ & $\begin{array}{l}E_{\text {cas }}> \\
1 \mathrm{TeV}\end{array}$ & $\begin{array}{l}E_{\text {cas }}> \\
100 \mathrm{TeV}\end{array}$ & Model \\
\hline \multirow[t]{4}{*}{ Unresolv. AGN } & 154 & 66 & $\begin{array}{ll}276 \\
\end{array}$ & 264 & Stecker et al. \\
\hline & 109 & 23 & 113 & 52 & Protheroe \\
\hline & 366 & 75 & 379 & 172 & Biermann \\
\hline & 897 & 148 & 680 & 125 & $\begin{array}{l}\text { Sikora \& } \\
\text { Begelmann }\end{array}$ \\
\hline NGC 4151 & 5.0 & 1.1 & - & - & Protheroe \\
\hline \multirow[t]{2}{*}{$3 \mathrm{C} 279$} & 20.3 & 4.2 & - & - & $\begin{array}{l}\nu=\gamma \\
\text { (extrap. from GRO) }\end{array}$ \\
\hline & 0.054 & 0.013 & - & - & Protheroe \\
\hline \multirow[t]{2}{*}{ Mkr-421 } & 3.2 & 0.67 & - & - & $\begin{array}{l}\nu=\gamma \\
\text { (obs. at } 1 \mathrm{TeV} \text { ) }\end{array}$ \\
\hline & 0.8 & 0.19 & - & - & Protheroe \\
\hline $3 \mathrm{C} 273$ & 0.8 & 0.19 & - & - & Protheroe \\
\hline $\operatorname{atm} . \nu$ & 2950 & 22.8 & 3435 & 5 & Volkova \\
\hline
\end{tabular}

Table 2. The number of muon and cascade events per year expected in DUMAND-II from unresolved and resolved AGNs, according to the various independent model calculations reported in proceedings of the 1992 Hawaii Workshop. Also shown are the rates expected from two sources observed by GRO, on the assumption that the neutrino flux equals the measured $\gamma$-ray flux (table taken from [Stenger 1992a, see there also for references).

The table also demonstrates the fact that the ratio of AGN neutrinos to atmospheric neutrinos improves with energy since the first have a harder spectrum than 
the latter. According to the Stecker model, an energy cut of $10 \mathrm{TeV}$ in the diffuse spectrum would have yield 66 AGN neutrinos on a background of 23 atmospheric neutrinos. With the much lower exclusion limits for diffuse fluxes of the year 2011, this cut value has moved to the region of some hundred $\mathrm{TeV}$.

The expected fluxes for individual AGN (with the exception of the GRO extrapolation for $3 \mathrm{C} 279$ ) are close or below the atmospheric background in a 2 degree search bin (defined by the angular resolution of about 1 degree at $1 \mathrm{TeV}$ ). This fact is reflected in the 1993 comparatively reluctant formulation of Halzen and Learned with respect to the capability of the telescopes to come in the mid-nineties.

\section{The community broadens}

\subsection{Surface detectors}

The high threshold required to get a detector working in a hostile environment such as the deep Pacific or the harsh conditions on the frozen Lake Baikal, resulted in apparently long preparatory periods of both DUMAND and Baikal. This led others to think about detectors near surface (for a review see [Belotti \& Laveder 1993]). The advantages seemed tempting: much easier access and a less challenging environment. Moreover, proven techniques like tracking chambers or Cherenkov techniques à la Kamiokande could be used. The author still remembers the insistence with which Masatoshi Koshiba, the father of Kamiokade, asked him in the mid nineties, at a meeting at the Jet Propulsion Laboratory /Pasadena: "Why don't you use the proven technique to see Cherenkov rings from tracks with the help of areas of phototubes like we do?" But the disadvantages were equally striking: the huge background from muons generated in cosmic rays showers, and the difficulty to expand these arrays much beyond a few $10^{4} \mathrm{~m}^{2}$. Actually, my answer to Koshiba focused to the latter fact. Several instruments were discussed in the late eighties and early nineties, most notably:

- GRANDE (Gamma-Ray and Neutrino DEtector), a dense phototube water Cherenkov array of about $30,000 \mathrm{~m}^{2}$ area at $0-50 \mathrm{~m}$ depth, to be installed in Arkansas Sobel 1988. GRANDE was proposed by H. Sobel, a leading scientist of the IMB proton decay detector (see section 6.2). It included twelve US and one Polish institution.

- LENA (Lake Experiment on Neutrino Activities), a large area water Cherenkov detector, also with a high coverage of the walls with PMTs and the aim to study neutrino oscillations with atmospheric and accelerator neutrinos and to do gamma and neutrino astronomy Nishikawa 1992.

- NET, a three layer water Cherenkov detector on top of Gran Sasso, essentially an Italian-French collaboration Bobisut 1991.

- PAN (Particle Astrophysics in Norrland), a Swedish initiative to deploy a shallow detector in one of the clear North-Swedish lakes, discarded later in favor of Swedish participation in AMANDA [Johannson 1991.

- SINGAO (Southern Italy Neutrino and Gamma Astronomy Observatory), a sampling array for air showers. $10,000 \mathrm{~m}^{2}$ of resistive plate chambers in the center were expected to be able separating upward going neutrino-induced muons from the huge background of downward muons Pistilli-1988. SINGAO was an Italy/UK collaboration.

None of these projects were realized, be it by financial reasons, by the failure to convincingly demonstrate the background rejection capabilities, or since shallow lake water parameters turned out to be worse than expected. However, the legacy of these 
projects can be found in two successful gamma detection arrays. That of the first four projects, all using Cherenkov detection, in the water pool detector MILAGRO in the US (now terminated and being replaced by the much bigger HAWC detector in Mexico); that of SINGAO in the resistive plate chamber detector ARGO/YBJ in Tibet. See for these detectors for charged cosmic rays and gamma rays the review articles of K.-H. Kampert and A. Watson Kampert \& Watson 2012 and E. Lorenz and R. Wagner Lorenz \& Wagner 2012.

\subsection{Underground detectors}

Whereas deep underwater and surface neutrino detectors were plagued with the problems described in the previous sections, some of the smaller underground detectors moved from success to success. Remarkably, two of these successes had not been on the top priority list of the experiments: neutrino oscillations (since the trust in their existence was low in the eighties) and neutrinos from supernova SN1987A (since Galactic or near-Galactic supernovae are rare). Table 3 lists the large underground experiments operating between 1978 and 1998 with high energy neutrino detection capability.

\begin{tabular}{|l|l|l|l|l|}
\hline Detector, Location & $\begin{array}{l}\mu \text { area } \\
\left(\mathrm{m}^{2}\right)\end{array}$ & Technique & Primary purposes & Operation \\
\hline \hline KGF, South India & 20 & ST & p-decay, atm. $\nu$ & $1980-1992$ \\
\hline Baksan, Caucasus & 250 & LS tanks & atm. $\nu$ SN & $1978-$ now \\
\hline IMB, Ohio & 400 & WC & p-decay & $1982-1991$ \\
\hline Kamiokande, Japan & 120 & WC & p-decay & $1983-1996$ \\
\hline NUSEX, French Alps & 10 & ST + Fe & p-decay & $1982-1998$ \\
\hline Frejus, Alps & 90 & GT $/$ ST+Fe & p-decay & $1984-1988$ \\
\hline Soudan I, USA & 10 & ST+concrete & p-decay & $1981-1990$ \\
\hline Soudan II, USA & 100 & DT+concrete & p-decay & $1988-2001$ \\
\hline MACRO, Gran Sasso & 1100 & LS + ST & monopoles, CRs & $1988-2001$ \\
\hline LVD, Gran Sasso & 800 & LS + ST & SN $\nu$ & 1992 now \\
\hline Super-K, Japan & 1000 & WC & p-decay, solar+atm. $\nu$ & $1996-$ now \\
\hline \hline
\end{tabular}

Table 3. Underground experiments with high energy capabilities, 1978-1998 (modified and updated from [Learned 1990]). WC = water Cherenkov, GT = Geiger Tubes, ST = streamer tubes, DT $=$ drift tubes, $\mathrm{LS}=$ liquid scintillator

The first massive detectors (1 kton or more) of the eighties were primarily constructed to detect proton decay, with a lifetime less than $10^{32}$ years, as predicted by early Grand Unified Theories. Proton decay was not found, instead three of the detectors recorded MeV-neutrinos from the supernova SN-1987A (Kamiokande 11 events, IMB 8 events and Baksan 5 events). Moreover, Kamiokande, together with the radio-chemical experiments ClAr (Homestake mine, USA), SAGE (Baksan laboratory) and GALLEX (Gran Sasso Laboratory) measured the solar neutrino flux. I refer to McDonald 2004 for an overview on solar and supernova neutrino experiments and to Bahcall 1994 for a compilation of historically relevant articles on solar neutrinos.

The solar neutrino data strongly supported the oscillation hypothesis, with the final confirmation by the SNO experiment in 2001 [Ahmad 2011. Neutrino oscillations denote the transformations between the three types ("flavors") of neutrinos: electron 
neutrinos $\left(\nu_{e}\right)$ muon neutrinos $\left(\nu_{\mu}\right)$ and tau-neutrinos $\left(\nu_{\tau}\right)$. They are only possible for massive neutrinos. According to this concept, the weak flavor eigenstates $\nu_{e}, \nu_{\mu}, \nu_{\tau}$ are linear combinations of mass eigenstates $\nu_{1}, \nu_{2}, \nu_{3}$. For the simplified case of two flavors $\nu_{\mu}, \nu_{\tau}$ and two mass eigenstates $\nu_{i}, \nu_{j}$ with different masses quantum mechanical time evolution of an initial $\nu_{\mu}$ state induces a non-zero transition probability to $\nu_{\tau}$. The survival probability for the muon neutrino is

$$
P\left(\nu_{\mu} \rightarrow \nu_{\mu}\right)=1-\sin ^{2}\left(2 \theta_{i j}\right) \cdot \sin ^{2}\left(\frac{1.27 \Delta m_{i j}^{2} \cdot L}{E_{\nu}}\right),
$$

where $L$ (in $\mathrm{km}$ ) is the distance traveled by the neutrino, $E_{\nu}$ (in $\mathrm{GeV}$ ) its energy and $\Delta m_{i j}^{2}=m_{i}^{2}-m_{j}^{2}\left(\right.$ in $\left.\mathrm{eV}^{2}\right)$. The three-flavour case is governed by two independent differences of mass squares and three mixing angles. The best-fit oscillation parameters derived from present data are:

$$
\begin{aligned}
& \left|\Delta m_{31}^{2}\right|=2.4 \times 10^{-3} \mathrm{eV}^{2} \approx\left|\Delta m_{23}^{2}\right| \quad \Delta m_{21}^{2}=7.65 \times 10^{-5} \mathrm{eV}^{2} \\
& \sin ^{2} 2 \theta_{23} \simeq 1 \quad \tan ^{2} \theta_{12}=0.304 \quad \sin ^{2} 2 \theta_{13} \sim 0.09
\end{aligned}
$$

The mass difference $\Delta m_{21}^{2}=7.65 \times 10^{-5} \mathrm{eV}^{2}$ has been derived from solar neutrino experiments. That the difference of the squared masses itself is known, rather than only its absolute value, is due to the fact that the neutrinos undergo not only the vacuum oscillations described by the formula above, but also matter induced, resonant oscillations in the Sun. These oscillations are sensitive to the mass difference and not only to its squared value.

Concerning the physics with neutrinos above a $100 \mathrm{MeV}$ (i.e. clearly above the energy of supernova burst neutrinos), the spectacular observation was the deficit of atmospheric neutrinos with increasing zenith angles. It pointed to the the second mass difference $\left|\Delta m_{31}^{2}\right|=2.4 \times 10^{-3} \mathrm{eV}^{2}$ which is due to muon neutrinos oscillating to tau neutrinos on their way through Earth. Interestingly this effect was controversial at the beginning.

The first detector which announced hints of oscillations of atmospheric neutrinos, i.e. a apparent deficit of atmospheric neutrinos, was the Baksan Neutrino Telescope BNT [Boliev 1981]. Given the uncertainties on the atmospheric neutrino flux and the methodical uncertainties of measurement, the deficit was not taken too seriously at that time. Actually, at the end of the eighties, BNT was among those underground experiments which declared no discrepancy between data and predictions. Later in the eighties, a deficit was reported by the water Cherenkov detectors IMB and Kamiokande but not by Frejus and NUSEX with their sandwiches of iron and tracking detectors. In the nineties, however, also tracking devices (Soudan-2 and MACRO) confirmed the effect. After a thorough study with Super-Kamiokande (with the first clear data showing muon neutrino rates as a function of zenith angle and energy, i.e. of $L / E$, see formula 3 , in 1998 it was eventually announced as discovery Fukuda 1998. The values of mass differences and mixing angles derived from underground experiments were subsequently confirmed and further iterated by experiments at nuclear reactors and accelerators and resulted in the values shown in (4) above.

Massive neutrinos provide the first hint for physics beyond the original standard model of particle physics. Moreover this phenomenon affects the predictions for neutrinos fluxes from astrophysical objects, starting with the Sun and ending with highenergy sources like Active Galactic Nuclei.

At the same time, neutrinos from high-energy astrophysics sources were not detected. Even the sky-maps from largest detectors with about $1000 \mathrm{~m}^{2}$ area (MACRO 
and Super-Kamiokande) did not show any significant excess over atmospheric neutrinos. In particular, none of the positions of notorious candidates such as Hercules-1, Geminga, the Crab Nebula, the binary systems SS-433 and Vela. Also the supernova SN-1987A did not show any indication of a high energy signal. It had been included according to the prediction of Berezinsky Berezinsky \& Priludsky 1977 that the ejecta of Supernovae (such as that of 1987) can be a strong source of neutrinos for months up to a few years.

Seen from today, the search for sources of high-energy neutrinos with detectors of $1000 \mathrm{~m}^{2}$ or less appears to have been hopeless from the beginning, with the possible exception of certain transient Galactic sources. But when these detectors had been constructed, this knowledge was not common and the search for point sources appeared as a legitimate (although not priority) goal.

\subsection{Neutrinos in ice?}

In this situation, a new, spectacular idea appeared on stage. In 1988, Francis Halzen from the University of Wisconsin gave a talk at the University of Kansas. At this occasion he was contacted by Ed Zeller, a Kansas glaciologist. Zeller told him about a small test array of radio antennas at the Soviet Vostok station, close to the geomagnetic South Pole. The Russians were going to test whether secondary particles generated in neutrino interactions could be detected via their radio emission. The idea that showers of charged particles would emit radio signals had been published back in 1962 by the Soviet physicist Gurgen Askaryan. Together with his colleagues Enrique Zas and Todor Stanev, Halzen realized that the threshold for this method was discouragingly high Halzen 1995. Instead he asked himself whether the optical detection via Cherenkov light, i.e. the DUMAND principle, would be also feasible for ice. In Halzen 1998] he remembers:

I suspect that others must have contemplated the same idea and given up on it. Had I not been completely ignorant about what was then known about the optical properties of ice I would probably have done the same. Instead, I sent off a flurry of E-mail messages to my friend John G. Learned, then the spokesman of DUMAND. ... Learned immediately appreciated the advantages of an Antarctic neutrino detector.

A few months later, Halzen and Learned released a paper "High energy neutrino detection in deep Polar ice" Halzen \& Learned 1988. With respect to the light attenuation length they proceeded

... on the hope that a simple test will confirm the belief that it is similar to the the observed $25 \mathrm{~m}$ attenuation length for blue to mid UV light in clear water in ocean basins.

Bubble-free ice was hoped to be found at depths smaller than $1 \mathrm{~km}$. Holes drilled into the ice and filled with water were supposed to refreeze or, alternatively, to be filled with a non-freezing liquid.

Learned sent, still in May 1988, a "notice of intent" to NSF, asking for support of a corresponding exploration program, but he got a negative response: it seemed obvious that he had his hands full with DUMAND. Halzen is a theorist, and so both did not proceed to do an experiment. But the idea made it to Buford Price's group at University of California, Berkeley. In 1989, two young physicists of the Price group, Doug Lowder and Andrew Westphal, joined a Caltech group drilling holes in Antarctic ice and tried to measure the ice transparency using existing boreholes. It would take, however, another year until the first successful transparency measurement 
of natural ice was performed - this time in Greenland. Bob Morse from the University of Wisconsin and Tim Miller (Berkeley) lowered photomultipliers into a $217 \mathrm{~m}$ hole drilled by glaciologists Lowder 1990].

In parallel to these first experimental steps, Buford Price, Doug Lowder and Steve Barwick (Berkeley), Bob Morse and Francis Halzen (Madison) and Alan Watson (Leeds) met at the International Cosmic Ray Conference in Adelaide and decided to propose the Antarctic Muon and Neutrino Detection Array, AMANDA.

In 1991 and 1992, the embryonic AMANDA collaboration deployed photomultipliers at various depth of the ice at the South Pole. Holes were drilled using a hot water drilling technique which had been developed by glaciologists. Judging the count rate of coincidences between photomultipliers (which are due to downgoing muons), the light absorption length of the ice was estimated at about $20 \mathrm{~m}$ and scattering effects were supposed to be negligible Morse 1993. It should turn out later, that this was a fundamental misinterpretation of the rates. But exactly this interpretation encouraged the AMANDA physicists to go ahead with the project.

\subsection{Neutrinos in the Mediterranean Sea?}

With ongoing activities in Hawaii and at Lake Baikal and the first ideas on a telescope in polar ice, the exploration of the Mediterranean Sea as a site for an underwater neutrino telescope was natural. First site studies along a route through the Mediterranean Sea were performed in 1989 by Russian physicists who also measured the muon counting rate as a function of depth Deneyko 1991. In July 1991, a Greek/Russian collaboration led by Leonidas Resvanis from the University of Athens performed a cruise and deployed a Russian built hexagonal structure made of titanium and carrying 10 photomultipliers down to a depth of $4100 \mathrm{~m}$. The site was close to Pylos at the West coast of the Peloponnesus. They measured the vertical muon intensity and the angular distribution of downgoing muons. This was the start of the NESTOR project [NESTOR, which was named after the mythic king of Pylos who counseled the Greeks during the Trojan war.

The advantages of the Pylos site were obvious: The depth can be chosen down to $5200 \mathrm{~m}$, dependent on the acceptable distance to shore, deeper than any other candidate site. This would reduce the background of downward muons. The water quality was excellent and the bio-luminescence seemed to be lower than at other Mediterranean sites.

In 1992, the collaboration included the University of Athens, the Scripps Institute for Oceanography in San Diego (USA), the Universities of Florence (Italy), Hawaii, Wisconsin (USA), Kiel (Germany) and the Institute for Nuclear Research Moscow. Naturally, more Greek institutes joined. Several French Universities joined in 1994 and left again in 1997 to pursue their own project ANTARES. More Italian groups from Rome and Florence joined in 1994, but in 1998 also decided to follow their own project, NEMO, close to Sicily. Since Italians had designed and constructed large parts of the off-shore electronics, this left the collaboration in a difficult state. Later, the Lawrence Berkeley National Laboratory, LBNL, got involved in the design of the electronics, profiting from parallel developments for IceCube. They provided essential electronics for the first stationary hexagonal floor which was deployed in 2004.

The results of the early cruises and the concept for the NESTOR detector were developed and presented during a series of Workshops in Pylos. NESTOR was conceived to consist of seven "towers" ( center) covering an area of about $10^{5} \mathrm{~m}^{2}$ as shown in Fig 10 .

A single tower should carry 168 PMTs on 12 hexagonal floors, vertically spaced by $20-30 \mathrm{~m}$, each with six omni-directional modules at the end of $16 \mathrm{~m}$ arms and one 


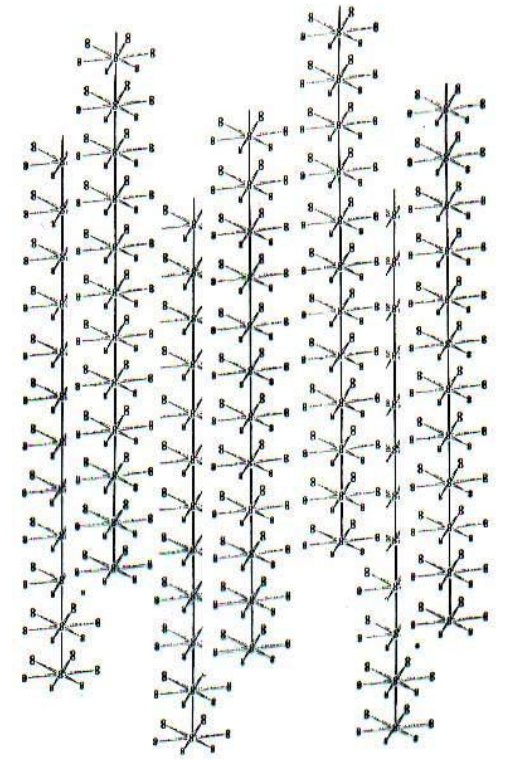

Fig. 10. The original NESTOR concept of a large array of towers, each carrying 12 floors of six arms. The optical modules are fixed at the end of the arms, plus one in the center. .

in the center Resvanis 1994. The "omni-directional module" contained two 15-inch PMTs, one upward, one downward looking, in two 17-inch glass pressure spheres.

The philosophy of NESTOR was to be not only sensitive to high energy neutrinos (therefore the large area covered by seven towers) but also to study atmospheric neutrino oscillations (hence the $5 \mathrm{GeV}$ threshold inside the geometrical volume of a tower and the omni-directionality of the modules).

It is noteworthy to recall that, similar to DUMAND, NESTOR had a strong initial activity on acoustic neutrino detection which was led by Igor Zheleznykh from INR Moscow. The acoustical array was named SADCO (Sea Acoustic Detection of Cosmic Objects), and was planned to consist of several hundred autonomous hydrophones spread over one cubic kilometer Karaevsky 1993. However, given the enormous challenges of NESTOR itself, the acoustic efforts went dormant after a while.

Nearly forgotten is another effort in the Mediterranean Sea, pursued by Peter Bosetti and students at Aachen Bosetti 1994. Their plan was to develop JULIA, a nested array, with an inner low-energy section (10 MeV threshold), moving to a layer tuned for $\mathrm{GeV}$ energies, and an outer envelope for the TeV-range. The preferred site was close to Rhodos, abouth $4 \mathrm{~km}$ deep. However, the second Gulf war prevented tests at this site. After a cruise to a site close to the Canaries (with participation of two Zeuthen scientists) and deployment of some equipment it became clear that manpower and funding were not sufficient to start an own project, and the project was terminated.

\section{The three-string race and the termination of DUMAND}

In 1993 and 1994, three collaborations were going to deploy detectors with three or more strings. Three strings are the minimum to achieve full spatial reconstruction of events.

The DUMAND collaboration was working towards installation of the first three of the nine DUMAND-II strings. Two of these strings were to be equipped with 
"Japanese Optical Modules" (JOMs) containing a 15-inch PMT "R2018" from Hamamatsu, and one string with "European Optical Modules" (EOMs) containing the hybrid XP2600 from Philips Wiebusch 1995. This stage was christened TRIAD.

In 1992, the AMANDA collaboration was joined by the Swedish collaborators from Stockholm and Uppsala. Steve Barwick, meanwhile at University of California in Irvine, designed a four-string detector with 80 PMTs which was going to be deployed between 800 and $1000 \mathrm{~m}$ depth. In Wisconsin, computer-controlled hot water drills were developed, in close collaboration with the Polar Ice Coring Office (PICO).

However, the first three-string array (NT36) was deployed at Lake Baikal in March/April 1993. It consisted of only 18 PMT-pairs at three strings of meager $40 \mathrm{~m}$ length. But it served its purpose to demonstrate that 3-dimensional reconstruction of muon tracks works as expected. John Learned from DUMAND recognized the importance of the Baikal achievement and sent an E-mail to the author: Congratulations for winning the 3-string race! Actually the first two neutrino candidates were isolated from the data taken with the same array in 1994 .

Meanwhile, in December 1993, a first string of the TRIAD, together with a string of environmental instruments was deployed and linked to shore via a junction box placed on the ocean bottom and a shore cable which had been laid a few months earlier. However, some pressure housings developed leaks. A short circuit in the junction box (the central component for communication to shore) did not clear due to a fuse failure, and soon the communication to shore failed 8 .

The DUMAND progress had been slow, but had shown remarkable progress compared to ocean research at that time. This impressed oceanographers but not the main funding organization, the Department of Energy (DOE), which was not used to a "try-and-try-again" mode of progress. Review committees without any ocean expertise judged the project, following criteria typical for accelerator research. Moreover, the termination of the Superconducting Super Collider (SSC) by the US congress in 1993 created a strong risk aversion in DOE. On the technical side, the reasons of the 1993 DUMAND failures had been identified and a redeployment was in preparation. But in 1995, the mentioned circumstances regrettably led to a termination of the support for DUMAND. From now on the goal to begin high energy neutrino astronomy was carried forward at the South Pole, in the Mediterranean Sea and in Lake Baikal.

\section{AMANDA}

AMANDA is located several hundred meters from the Amundsen-Scott station. Holes of $60 \mathrm{~cm}$ diameter were drilled with pressurized hot water; strings with optical modules were deployed in the water which subsequently refreezes. Installation operations at the South Pole are performed in the Antarctic summer, November to February. For the rest of the time, two operators (of a winter-over crew of 25-40 persons in total) maintained the detector, connected to the outside world via satellite communication.

Already in 1990 in Greenland, the physicists were assisted by Bruce Koci who was working for the Polar Ice Core Office (PICO). He then became the "drill master" of AMANDA. Without Koci, AMANDA would certainly not have developed so fast and smooth as it did. "The success of AMANDA rested on two pillars: the Amundsen Scott Station and Bruce Koci." - as Francis Halzen formulated when Koci deceased in 2006.

The first AMANDA array with 80 optical modules on four strings was deployed in the austral summer 1993/94, at depths between 800 and $1000 \mathrm{~m}$ Askebjer 1995. Surprisingly, light pulses sent from one string to a neighbored string over $20 \mathrm{~m}$ distance

\footnotetext{
8 Deployment, string recovery and initial problem analysis are summarized in Grieder 1994
} 
did not arrive after the expected $100 \mathrm{~ns}$, but were considerably delayed. The surprise was resolved at the 1994 Venice Workshop on Neutrino Telescopes. Here, Grigorij Domogatsky informed Francis Halzen about results from an ice core extracted at the geomagnetic South Pole where the Russian Vostok station is located. The data demonstrated, that air bubbles which remain from the original firn ice at the surface did not yet disappear at $1 \mathrm{~km}$ depth. The delay was due to light scattering on the bubbles. Light would not travel straight but via random walk and become nearly isotropic after a a few times the distance called effective scattering length. The effective scattering length was found to be between $40 \mathrm{~cm}$ at $830 \mathrm{~m}$ depth and $80 \mathrm{~cm}$ at $970 \mathrm{~m}$. The scattering by air bubbles trapped in the ice made track reconstruction impossible.

A great story might have been over before it really got started. AMANDA seemed to be "nothing other than a big calorimeter" - as Leonidas Resvanis sarcastically commented - without any real tracking capabilities. This could have been the point to give up the project. Nevertheless, the group from DESY joined. We were encouraged by a trend seen in the AMANDA data itself, as well as by ice core data taken at the Russian Vostok station: below 1300 meters bubbles should disappear.

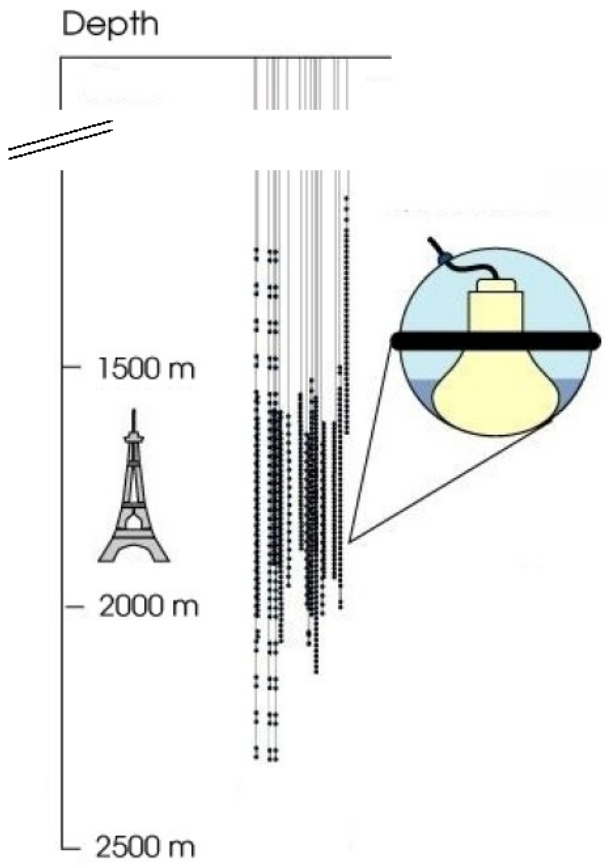

Fig. 11. The AMANDA configuration. The detector consisted of 677 optical modules at 19 strings. Three of the strings have been sparsely equipped towards larger and smaller depth in order to explore ice properties, one string got stuck during deployment at too shallow depth and was not used in analyses. The Eiffel tower is shown to scale for size comparison.

This expectation was confirmed with a second 4-string array which was deployed in 1995/96. The remaining scattering, averaged over 1500-2000 $\mathrm{m}$ depth, corresponds to an effective scattering length of about $20 \mathrm{~m}$ and is assumed to be due to dust. This is still considerably worse than for water but sufficient for track reconstruction Ackermann 2006. The proof that it indeed was sufficient took some time, as well as the development of the suitable reconstruction methods and selection criteria. The identification of the first two neutrino events from the data taken with the 4-string configuration(1996) is described in Andres 1999], the sophisticated reconstruction and selection methods in Ahrens 2004. The average absorption length at $400 \mathrm{~nm}$ is about $100 \mathrm{~m}$, much larger than at water detector sites. The array was upgraded 
stepwise until January 2000 and eventually comprised 19 strings with a total of 677 optical modules, most of them at depths between 1500 and $2000 \mathrm{~m}$. Figure 11 shows the final configuration of the AMANDA array.

In Fig. 12, absorption and scattering coefficients are shown as functions of depth and wavelength Ackermann 2006. The variations with depth are due to bubbles at shallow depth leading to very strong scattering and, at depths greater than $1400 \mathrm{~m}$, to dust and other material transported to Antarctica during varying climate epochs. The quality of the ice improves substantially below a major dust layer at a depth of about 2000-2100 m, with a scattering length about twice as large as for the region above $2000 \mathrm{~m}$. The depth dependence of the optical properties complicates the analysis of the experimental data. Furthermore, the large delays in photon propagation due to the strong scattering cause worse angular resolution of deep-ice detectors compared to water. On the other hand, the large absorption length, with a cut-off below $300 \mathrm{~nm}$ instead of $350-400 \mathrm{~nm}$ in water, results in better photon collection.
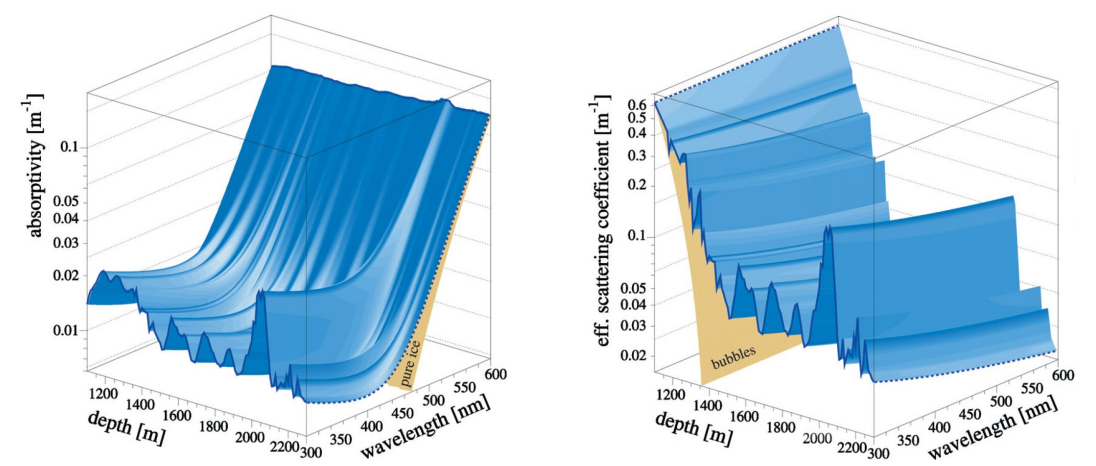

Fig. 12. Absorption coefficient (left) and scattering coefficient (right) in the South Polar ice as functions of depth and wavelength Ackermann 2006.

The relatively short distance between optical modules and surface electronics allowed for transporting the analogue signals of the photomultipliers to surface over $2 \mathrm{~km}$ of cable instead of digitizing them in situ. This requires a large output signal of the photomultiplier, a specification met by the 8-inch R5912-2 from Hamamatsu with 14 dynodes and a gain of $10^{9}$. The first ten strings used copper cables for both high-voltage supply and signal transmission; for the last 9 strings the anode signal was used to drive a LED, and the light signal was transmitted to surface via optical fibers. The feasibility of transmitting analog signals via $2 \mathrm{~km}$ optical fibers was all but obvious at the beginning. The idea is due to Albrecht Karle, at that time postdoc at DESY. It was later also used in the MAGIC gamma telescope at the Canary Islands (although via shorter distances). The time resolution achieved was better than $5 \mathrm{~ns}$; given the strong smearing of photon arrival times due to light scattering in ice, this jitter appeared to be acceptable. For optical fiber transmission the time resolution was slightly better than for copper cable, as was the dynamic range.

A big advantage of deep ice compared to deep water is the small optical noise rate, about $0.5 \mathrm{kHz}$ in an 8 -inch tube, compared to $20-60 \mathrm{kHz}$ due to $\mathrm{K}^{40}$ decays and bio-luminescence in lakes and oceans. The contamination of hit patterns from particle interactions with noise hits is thus small and makes hit selection much easier than in water and allows identifying burst-like low-energy events from Supernovae (see section 10.

The angular resolution of AMANDA for muon tracks was $2^{\circ}-2.5^{\circ}$, with a lower energy threshold around $50 \mathrm{GeV}$. Although better than for Lake Baikal $\left(3^{\circ}-4^{\circ}\right)$, this 
was much worse than for ANTARES $\left(<0.5^{\circ}\right.$, see below $)$. This is the result of the strong light scattering which deteriorates the original information contained in the Cherenkov cone.

AMANDA was switched off in April 2009, after more than 9 years of data taking in its full configuration. Figure 13 shows the skyplot derived from of 6959 neutrinos taken in the years 2000-2007. None of the spots is statistically significant, therefore only upper limits could be derived.

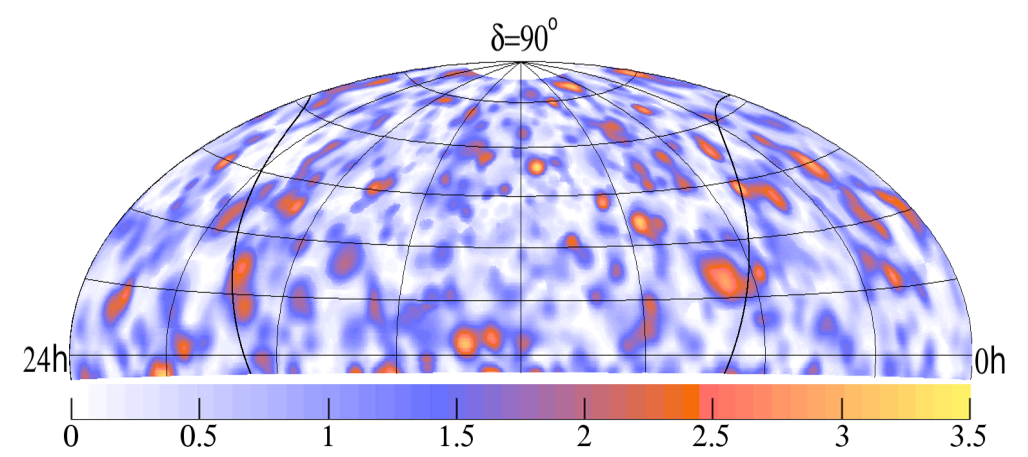

Fig. 13. 7-year significance map of the Northern hemisphere derived with AMANDA Abbasi 2009.

AMANDA provided record limits on fluxes for cosmic neutrinos, be it for diffuse fluxes, for point sources or for transient sources like Gamma Ray Bursts. These limits ruled out the first models on neutrino production in cosmic sources.

The AMANDA limits on the diffuse neutrino flux have to be seen in the context of the theoretical models and bounds existing at that time. The commonly accepted "benchmark" bound was been derived from charged cosmic ray fluxes ("WaxmanBahcall bound" Waxman \& Bahcall 1999]) and normalised to the cosmic ray flux at about $10^{19} \mathrm{eV}$. Assuming a generic $E^{-2}$ spectrum for all extragalactic sources, the authors obtained a limit of $E^{2} \cdot d N / d E^{2}=1-5 \times 10^{-8} \mathrm{GeV} \mathrm{cm}^{-2} \mathrm{~s}^{-1} \mathrm{sr}^{-1}$, with the uncertainty given by different cosmic evolution models. This estimate assumed that the sources are sufficiently dilute, so that neutrons can escape, decay and provide the observed cosmic rays to which the estimate is normalised. If the sources are opaque even for neutrons, the only remaining estimator is electromagnetic radiation. The decay of $\pi^{0} \mathrm{~s}$ (co-produced with charged pions producing neutrinos) yields gamma rays. Those develop electromagnetic cascades leading to gamma rays of lower energies. Measurements of gamma rays in the $\mathrm{MeV}-\mathrm{GeV}$ energy range from a satellite had set a bound to the neutrino flux, which hardly could be circumvented by more sophisticated assumptions on the character of the sources. This "gamma limit" (first introduced in Berezinsky \&Smirnov 1975]) was close to a second bound derived from cosmic ray spectra: Contrary to Waxman and Bahcall, the authors Mannheim, Protheroe and Rachen (MPR) [Mannheim 1998] assumed that a significant part of the observed cosmic ray spectrum below $10^{19} \mathrm{eV}$ was due to extragalactic rather than Galactic sources. Interpreting the cosmic ray spectrum between $10^{16} \mathrm{eV}$ and $10^{19} \mathrm{eV}$ essentially as a superposition of spectra from many extragalactic source classes, each with a different cut-off, the neutrino bound considerably weakened to $E^{2} \cdot d N / d E \approx 5 \times 10^{-7}$ $\mathrm{GeV} \mathrm{cm}^{-2} \mathrm{~s}^{-1} \mathrm{sr}^{-1}$ at a few $10^{14} \mathrm{eV}$. AMANDA could exclude the MPR limit, but the exclusion of the WB limit was only achieved with IceCube (see section 11).

AMANDA extended the measured spectrum of atmospheric neutrinos by nearly two orders of magnitude, from a few $\mathrm{TeV}$ to $200 \mathrm{TeV}$ (Fig.14). It also established 
record limits on indirect dark matter search, on the flux of magnetic monopoles, and on effects violating Lorentz invariance (see for a summary of results and references Katz \& Spiering 2011). It would have detected neutrinos from a supernova burst in our Galaxy (if such a burst would have appeared!), and it provided results on the spectrum and composition of cosmic rays.

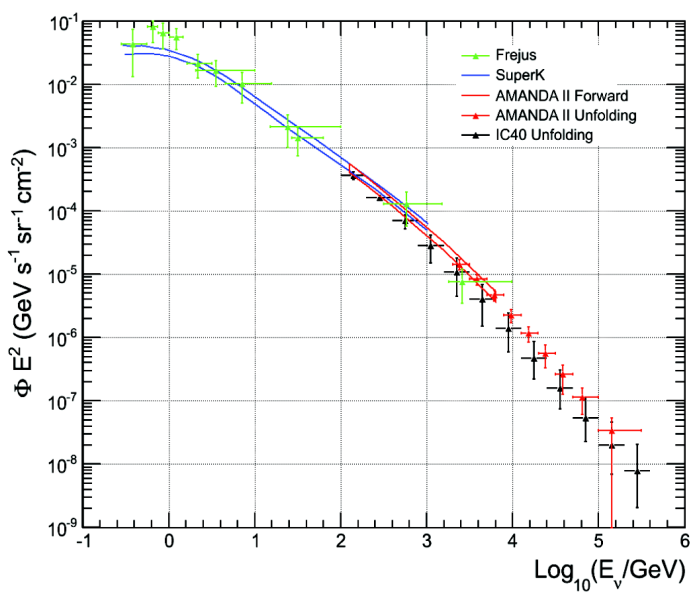

Fig. 14. Energy spectrum of atmospheric neutrinos Abbasi 2011.

What was hoped for in optimistic dreams - the discovery of an extraterrestrial source of neutrinos - did not happen. But there was one moment when the adrenaline level of some of us went up and we thought we were close to a discovery. While analyzing in 2005 the data taken from 2000-2003, five events where identified from the direction of the Active Galaxy 1ES1959+650. Interestingly, three of these came within 66 days in 2002 Ackermann 2006a. Two of the three neutrinos were coinciding within about a day with gamma-ray flares observed by the gamma-ray telescopes HEGRA and Whipple - see Fig15. Excitingly, one of these two flares was not accompanied by an X-ray flare, a so-called "orphan flare", which one would expect for a hadron flare where the X-ray flux accompanying electron flare is absent. This result was quickly followed by two theoretical papers, one claiming that the corresponding neutrino flux would not fit any reasonable assumption on the energetics of the source Reimer 2005, the other claiming that scenarios yielding such fluxes were conceivable Halzen \& Hooper 2005. Since the analysis was not a fully blind analysis, it turned out to be impossible to determine chance probabilities for this event, and actually the result was never published in a journal. However, it initiated considerations to send alerts to gamma-ray telescopes in case time-clustered events from a certain direction would appear. Such a "Target-of-Opportunity" alert is currently operating between IceCube and the gamma-ray telescopes MAGIC (La Palma) and VERITAS (Arizona).

\section{Mediterranean Sea: NESTOR, ANTARES, NEMO}

Whereas AMANDA and Baikal developed coherently, the Mediterranean community split in three branches which are related to three different locations and host countries.

The NESTOR collaboration presented the full concept of a "tower" in 1993 Resvanis 1994. After a long phase of tests and developments, a cable was installed to a site at $4 \mathrm{~km}$ depth. In 2004, a single prototype floor was deployed, connected 


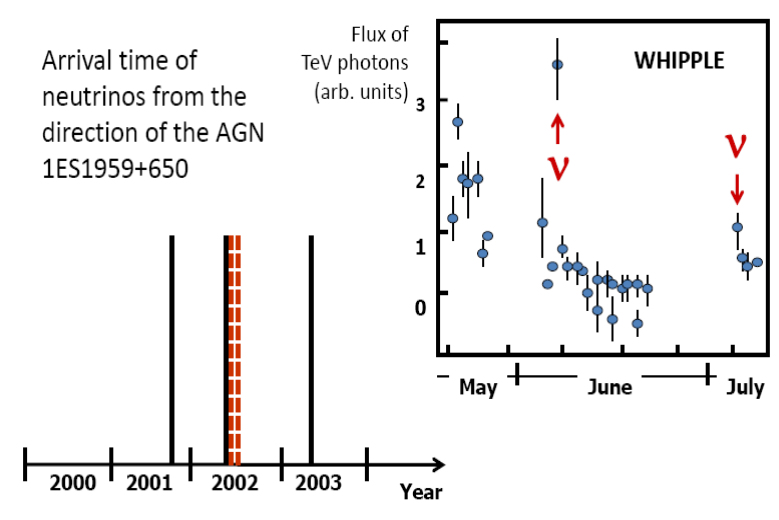

Fig. 15. The "curious" coincidence of neutrino events from the direction of an AGN with gamma flares from the same source. The second and third of the three events recorded in 2002 (dashed) coincide within about one day with peaks seen by WHIPPLE.

and operated for about one month Aggouras 2005. Then, its operation had to be terminated due to a failure of the cable to shore. However, the data taken with this prototype demonstrated the detector functionality and provided a measurement of the atmospheric muon flux Aggouras 2005a.

Comparing the original plan to deploy an array of towers within a decade (see Fig 10 with the reality of a single floor operated over just a month, demonstrates the enormous challenges which these projects face. The deep sea medium is hostile and unforgiving, the iterative approach is not what funding agencies like. A professional management and a coherent collaboration are necessary for any large projects, and if problems from this corner add to the inherent problems of deep-underwater projects, delays or even failure are inevitable.

Currently NESTOR is part of the KM3NeT framework which is directed towards a multi-cubic kilometer detector in the Mediterranean Sea.

French collaborators who temporarily had been members of NESTOR, pursued an independent strategy from the mid-nineties. Together with collaborators from Italy and the Netherlands they presented a full proposal for a 12-string detector in 1999 Aslanides 1999. In 2001 also a German group at the University Erlangen joined the experiment. ANTARES stands for Astronomy with a Neutrino Telescope and Abyss environmental RESearch) ANTARES. This proposal was based on the operation of a demonstrator string Blondeau 1998 Feinstein 1999 as well as on the results of extensive site exploration campaigns in the region off Toulon at the French Mediterranean coast, indicating that the optical background Amram 2000 as well as sedimentation and biofouling Amram 2003 are acceptable at that site. However, taken all together (depth, optical clarity, optical background and sedimentation), the site is inferior to the Greek and Italian sites.

The construction of ANTARES started in 2002 with the deployment of a shore cable and a junction box, the central element connecting the shore cable to the detector. In 2002/2003, a preproduction string was deployed and operated for a few months. Several technical problems were identified that required further studies, design modifications and the operation of a mechanical test string Ageron 2007. The detector in its final 12-string configuration was installed in 2006-2008 and has been operational since then, with a break of a few months in 2009 due to a failure of the main cable that required repair.

ANTARES consists of 12 strings, each carrying 25 "storeys" equipped with three optical modules, an electronics container and calibration devices. The optical mod- 


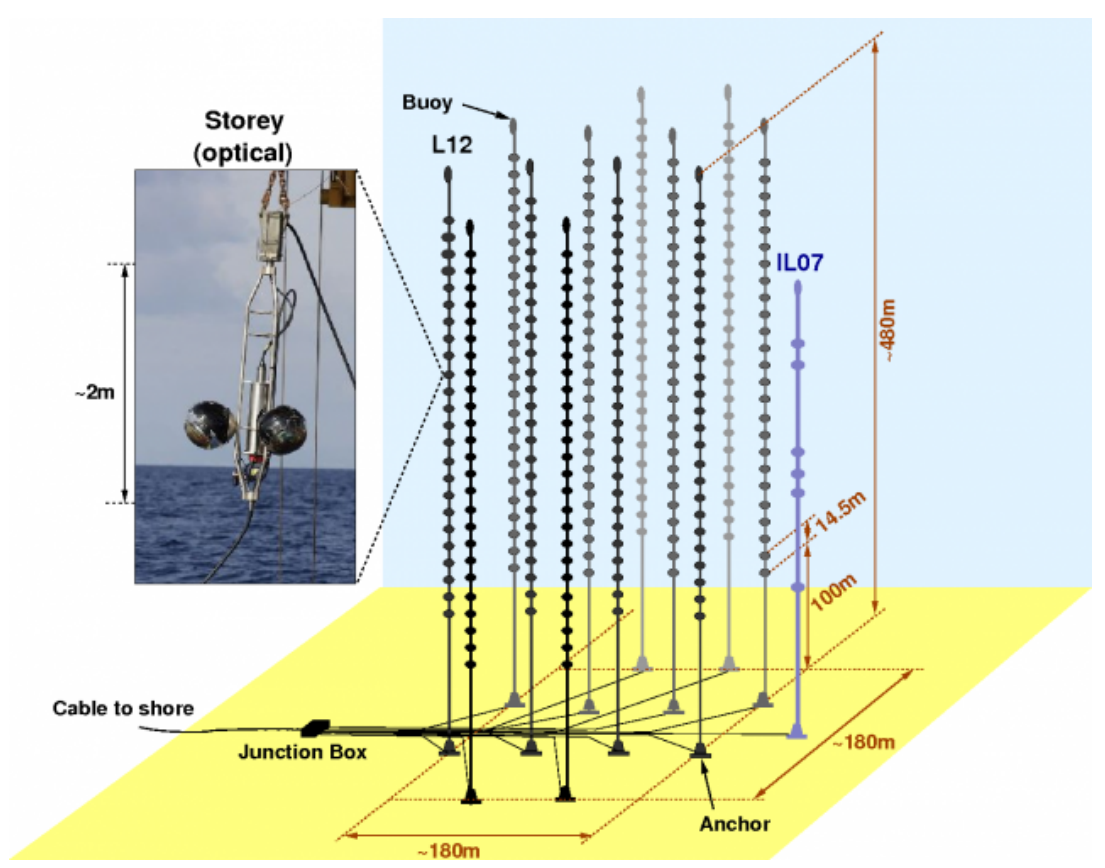

Fig. 16. Schematic of the ANTARES detector. Indicated are the 12 strings and the instrumentation line in its 2007 configuration (IL07). Shown as an inset is the photograph of a storey carrying 3 photomultipliers.

ule consists of a 17-inch glass sphere housing a hemispherical 10-inch photomultiplier (Hamamatsu R7081-20). A further string, the "instrumentation line", carries devices for environmental monitoring. The depth at the ANTARES site is $2475 \mathrm{~m}$. The schematic setup is shown in Fig. 16, a detailed technical description can be found in Ageron 2011.

An almost background-free separation of neutrino-induced upward-going muons from the huge background of downward-going muons is the central requirement for an underwater or under-ice telescope. Baikal and AMANDA, followed by ANTARES, have quickly mastered this challenge, even more so IceCube. Figure 17(left) shows the rate of muons as a function of the zenith angle $\theta$ as measured with ANTARES. Below the horizon $(\theta<0)$ the rate is well described by the expectation for atmospheric neutrinos, above the horizon by that for atmospheric muons. The right side of the figure shows the 1-year skymap of neutrino candidates - as to be expected without a clear source signal.

The newest Mediterranean project is NEMO (NEutrino Mediterranean Observatory) NEMO. It was launched in 1998 after Italian groups left NESTOR. After it had turned out that the original goal to deploy a tower before the end of 1997 was not realistic, they had counted on a test set-up in the shallow water of the Navarino Bay not far from the NESTOR shore station. But further delays led to the termination of Italian NESTOR participation in 1998.

In the same year the Italians started to discuss a possible continuation of Cherenkov underwater neutrino astronomy without the frictions they felt the years before. Antonino Capone, the chair of the Rome group, was contacted by Emilio Migneco from Lab. Nazionale del Sud (LNS) in Catania, Sicily. As a result, Italians started activities for the characterization of deep-sea sites close to the Italian Mediterranean coasts. 

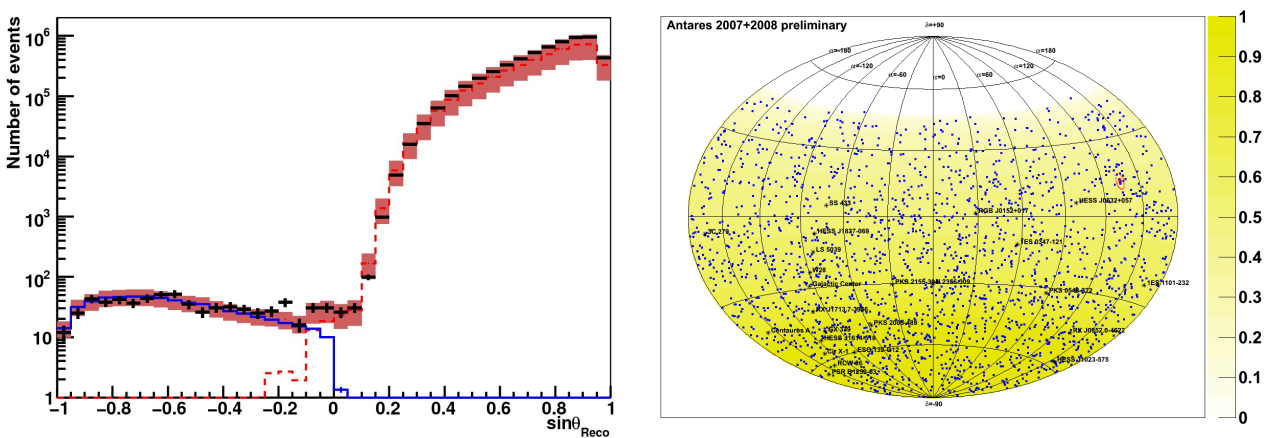

Fig. 17. Left: Number of reconstructed muons in the 2008 ANTARES data, as a function of the reconstructed zenith angle (black error bars). Also indicated are the simulation results for atmospheric muons (red dashed), and muons induced by atmospheric neutrinos (blue). The shaded band indicates the systematic uncertainties. Figure taken from Aguilar 2011. Right: Equatorial skymap of neutrino-induced muon events from 295 days of ANTARES data in $2007 / 2008$. The background color scale indicates the sky visibility in percent of the time. The most significant accumulation of events, marked with a red circle, is fully compatible with the background expectation [Eberl 2011].

Migneco presented the NEMO R\&D project to the corresponding INFN Board and the project started, with groups from Bari, Catania, Frascati, Messina and Roma. Still in 1998, first sea campaigns followed and studied various sites close to Italy.

From the beginning, the objective of this project was to study the feasibility of a cubic kilometer detector, to develop corresponding technologies and to identify and explore a suitable site, in this case close to Sicily, rather than to build an separate detector of medium size. Therefore it is no surprise that in 2000 the Italian NEMO members joined ANTARES, in parallel to their own activities directed to the cubic kilometer scale.

The basic unit of NEMO are towers composed by a sequence of floors. Different to NESTOR, floors consist of horizontal "bars", originally foreseen to be $15 \mathrm{~m}$ long and each equipped with four 10-inch PMs. The floors are tilted against each other and form a three-dimensional structure. Capone 2009] (see Fig. 18]. A tower can be folded together and deployed to the sea floor as a compact object that is subsequently unfurled. Contrary to single strings and similarly to the NESTOR concept, the 3dimensional arrangement of photomultipliers per tower allows for local reconstruction of muon directions.

The most suitable site at a depth of $3.5 \mathrm{~km}$, about $100 \mathrm{~km}$ off Capo Passero on the South-Eastern coast of Sicily has been identified and investigated during various campaigns. During the first prototyping phase, a cable to a test site near Catania at a depth of $2 \mathrm{~km}$ was installed and equipped with a junction box. In 2007, a "mini-tower" with 4 bars was deployed, connected and operated for several weeks. Although the data taking period was limited to a few months due to technical problems, the minitower provided the proof of concept for the technologies and most of the components employed. The flux of atmospheric muons was determined in good agreement with the expectations from simulation Aiello 2010.

The setup of a second phase Taiuti 2011] includes shore infrastructure at Capo Passero and a $100 \mathrm{~km}$ long cable to the site at $3.5 \mathrm{~km}$ depth; both are currently in place. A remotely operated vehicle (ROV) is available for the deep-sea operations. A mechanical test tower of limited size was successfully deployed and unfurled in early 


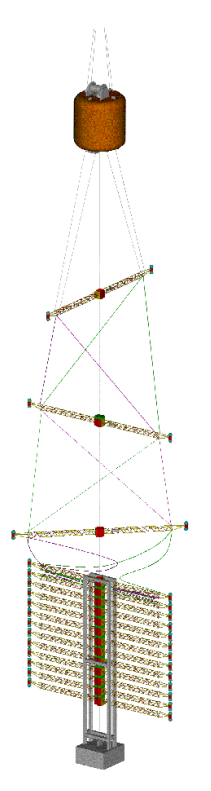

Fig. 18. Sketch of the principle of a flexible NEMO tower during the unfurling process. Note that exact configuration and the packaging of the bars does not correspond to the current design framework.

2010. The plans to deploy a full-size prototype tower will be pursued in the KM3NeT framework.

\section{IceCube}

IceCube IceCube is located at the South Pole and actually incorporates its predecessor AMANDA as a sub-array (see Fig. 19. With this detector, the idea of a cubic-kilometer detector was finally realized. However, the path towards the first installation was all but smooth.

Actually, the first initiative beyond AMANDA was a concept called DeepIce, a proposal for multidisciplinary investigations, including neutrino and cosmic ray astrophysics, glaciology, glacial biology, seismology and climate research. As an example, we note the relation between the layered impurities from dust and climatic effects or volcano eruptions Ackermann 2006. DeepIce was proposed in 1999 to NSF, but was not funded: Several of the referees concluded that a neutrino detector was sold under the flag of multi-disciplinary research, (mis) using the NSF funding model for multidisciplinary centers. The advice was to go ahead with a dedicated project for a neutrino telescope.

As a consequence, already in November of the same year a first 67-page IceCube proposal was submitted to NSF IceCube 1999. It had been worked out essentially by the collaborators of the old AMANDA collaboration. i.e. the US Universities in Atlanta, Baton-Rouge, Berkeley, Delaware, Irvine (Univ. of California), Kansas, Madison, Philadelphia (Pennsylvania Univ.) and IAS Princeton and LBNL Berkeley. Germany participated with DESY and the Universities Mainz and Wuppertal, Sweden and Belgium with the Universities Brussels, Stockholm and Uppsala. Soon, a number of additional institutions became interested and a new collaboration was formed, the IceCube collaboration, which meanwhile has grown to nearly 40 institutions. Paradoxically, the two collaborations co-existed until 2005 then joining to one collaboration, IceCube.

Naturally, the mixture of AMANDA pioneers and the new members did not go without some tensions. Some AMANDA pioneers feared the newcomers would ig- 
nore the merits of AMANDA, some new IceCube members suspected that AMANDA colleagues would look too much back and not forward. These tensions were exemplified by a hot debate on the best concept for data transmission. A group headed by DESY favored analog transmission via optical fibers, another group headed by LBNL preferred digital transmission. The first solution was proven to be feasible in AMANDA but suffered from the vulnerability of optical fibers and connectors and a rather difficult calibration procedure, the second solution was standard in accelerator experiments, appeared to be more modern, but there were doubts whether the longterm reliability and the timing accuracy could be as good as promised. The digital concept itself did not come as a total novelty: the 18th string of AMANDA had been equipped, in addition to the analog readout, with a digital readout which incorporated all basic elements of what was later used in IceCube Ackermann 2006b. In 2001, the controversy was decided following the recommendation of an external expert panel led by Barry Barish, which prioritized the digital transmission. In the mean time we definitely know that this was the right decision. With its fantastic reliability and stable 2 ns timing accuracy, the DOM concept (DOM for Digital Optical Module) has exceeded all expectations.

The IceCube Technical Office is located at the University of Wisconsin, with project director Jim Yeck. Under the control of the Technical Office, IceCube components were developed in various labs of the collaboration. The DOM was developed in LBNL, the communication card to the DOMs from surface in DESY. The integrated design, the HV system and a "LED flasher board" were developed in Wisconsin. Assembly and testing of the DOMs was performed at three locations, in Wisconsin, in Stockholm/Uppsala and in Zeuthen.

For IceCube construction, the thermal power of the hot-water drill factory was upgraded to $5 \mathrm{MW}$, compared to $2 \mathrm{MW}$ for AMANDA. This reduced the average time to drill a $2450 \mathrm{~m}$ deep hole with a diameter of $60 \mathrm{~cm}$ to 35 hours. The commissioning of the drill during the first deployment season 2004/05 turned out to be extremely challenging, but eventually a first, single string was deployed in January 2005: The first step was made! The following seasons resulted in 8, 13, 18, 19, 20 and 7 strings, respectively. The last of 86 strings was deployed at Dec. 18, 2010.

IceCube consists of 5160 digital optical modules (DOMs) installed on 86 strings at depths of 1450 to $2450 \mathrm{~m}$. A string carries 60 DOMs with 10-inch photomultipliers Hamamatsu R7081-02 housed in a 13-inch glass sphere. Signals are digitized in the DOM and sent to the surface via copper cables. 320 further DOMs are installed in IceTop, an array of detector stations on the ice surface directly above the strings (see Fig. 19). AMANDA, initially running as a low-energy sub-detector of IceCube, was decommissioned in 2009 and replaced by DeepCore, a high-density sub-array of eight strings at large depths (i.e. in the best ice layer) at the center of IceCube. DeepCore collects photons with about six times the efficiency of full IceCube, due to its smaller spacing, the better ice quality and the higher quantum efficiency of new PMTs. Together with the veto provided by IceCube, this results in an expected threshold of about $10 \mathrm{GeV}$. This opens a new window for oscillation physics and indirect dark matter search.

The muon angular resolution achieved by present reconstruction algorithms is about $1^{\circ}$ for $1 \mathrm{TeV}$ muons and below $0.5^{\circ}$ for energies above $10 \mathrm{TeV}$. Unlike underwater detectors with their environment of high optical noise, IceCube can be operated in a mode that is only possible in ice: The detection of burst neutrinos from supernovae. The low dark-count rate of the PMTs allows for detection of the feeble increase of the summed count rates of all PMTs during several seconds, which would be produced by millions of interactions of few-MeV neutrinos from a supernova burst Abbasi 2011a. IceCube records the counting rate of all PMTs in millisecond steps. A supernova in the center of the Galaxy would be detected with extremely high significance and the 


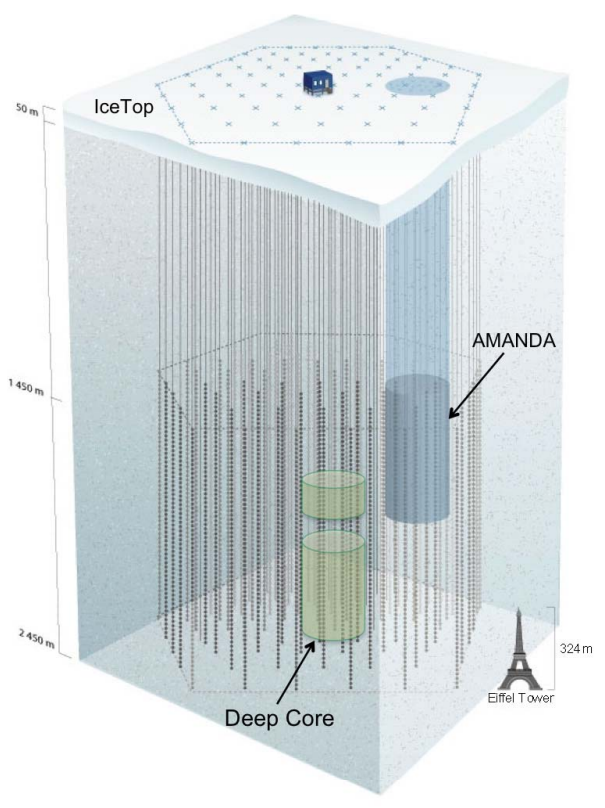

Fig. 19. Schematic view of the IceCube neutrino observatory. AMANDA was replaced by DeepCore, a nested lowthreshold array. At the surface, the air shower array IceTop and the IceCube counting house are indicated.

onset of the pulse could be measured in unprecedented detail. Even a SN 1987A-type supernova in the Large Magellanic Cloud would provide a recognizable signal and be sufficient to provide a trigger to the SuperNova Early Warning System, SNEWS Antonioli 2004.

\section{Where do we stand?}

With IceCube, the sensitivity to point sources and to diffuse fluxes has been improved by nearly a factor of thousand when compared to the situation of the mid nineties. But alas - no indication for extraterrestrial sources has found yet, but only ever tightening upper limits on fluxes have been established.

Figure 20 compiles the limits from previous experiments, from the different IceCube stages and from ANTARES. Note that the combined data of IceCube-40 and IceCube-59 surpass the mark of $1 \mathrm{~km}^{3} \times 1$ year and thus exceed 1 year worth of data from the full IceCube detector. When this article is printed, a factor of 1000 improvement of the sensitivity to point sources will have been reached when compared to the very first AMANDA point source paper from the year 2000 Andres 2000. Searching for coincidences of neutrinos with Gamma Ray Bursts reported by satellites, IceCube also challenges the hypothesis that Gamma Ray Bursts are the sole origin of cosmic rays of highest energies Abbasi 2012.

At the same time, predictions for neutrino fluxes from extraterrestrial sources went lower and lower. This is best demonstrated by Figure 21. It shows the number of events per year in a cubic kilometer detector expected from the supernova remnant RX J1713-3946, the most preferred galactic candidate source.

The drop from the 2002 paper of Alvarez-Muniz and Halzen (shortly before the construction of IceCube started) to the paper of Costantini and Vissani from 2005 is due to that fact that in the first paper oscillations have not been taken into account 


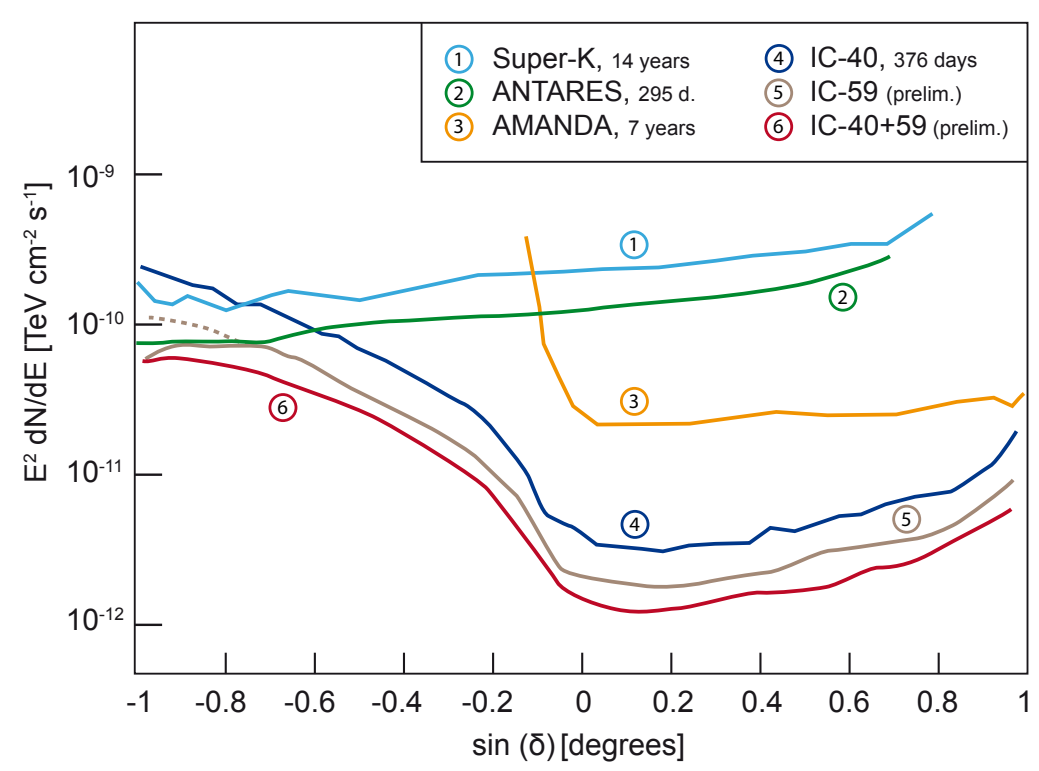

Fig. 20. Point source limits from various experiments. See Katz \& Spiering 2011 for references.

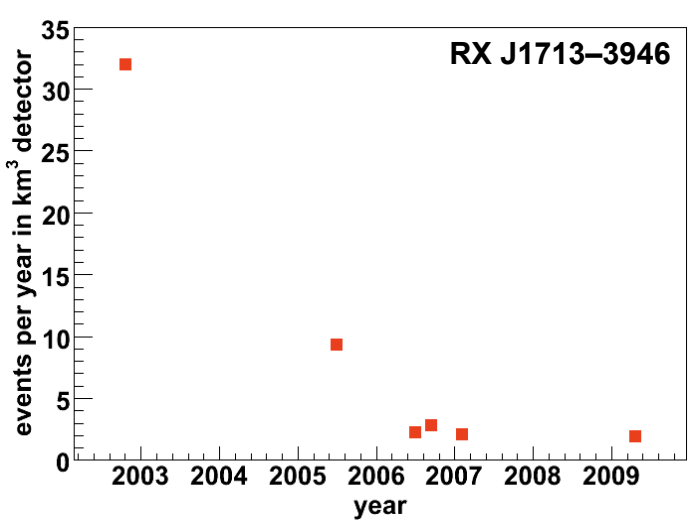

Fig. 21. Number of events per year in a cubic kilometer detector expected from the supernova remnant RX J1713-3946 Predictions are shown for Alvarez-Muniz \& Halzen 2002, Costantini \& Vissani 2005, Distefano 2006 Kistler\&Beacom 2006, Kappes 2007 and Morlino 2009. Courtesy of Alexander Kappes.

9 and that better gamma-ray data on the source were available. The following reduction is due to further improved data and more realistic calculations. After 2005 the predictions stabilize, however on a disappointingly low level: with 2-3 events per year on a much larger background of atmospheric neutrinos, a significant detection would need more than ten years of data taking. Note that even this is an optimistic assumption, since all the calculations assume the the gamma-rays observed from this source are due to the decay of $\pi^{0}$ s from hadronic interactions (with charged pions decaying to neutrinos as counterparts), and not to inverse Compton scattering $e^{-}+\gamma_{\text {low energy }} \rightarrow e^{-}+\gamma_{\text {high energy }}$.

\footnotetext{
${ }^{9}$ Oscillations turn the original ratio $\nu_{e}: \nu_{\mu}: \nu_{\tau}=1: 2: 0$ to $\nu_{e}: \nu_{\mu}: \nu_{\tau}=1: 1: 1$, thereby reducing the number of muon neutrinos as the only neutrino suitable for point source searches by a factor of 2. See also the paper of Learned and Pakvasa Learned \& Pakvasa 1995] where the authors propose to search for "double-bang" events for tau-neutrino identification.
} 
Point-source searches use the directional and energy information to reduce the background from atmospheric neutrinos. Cosmic neutrinos from a given source would cluster around the source direction.

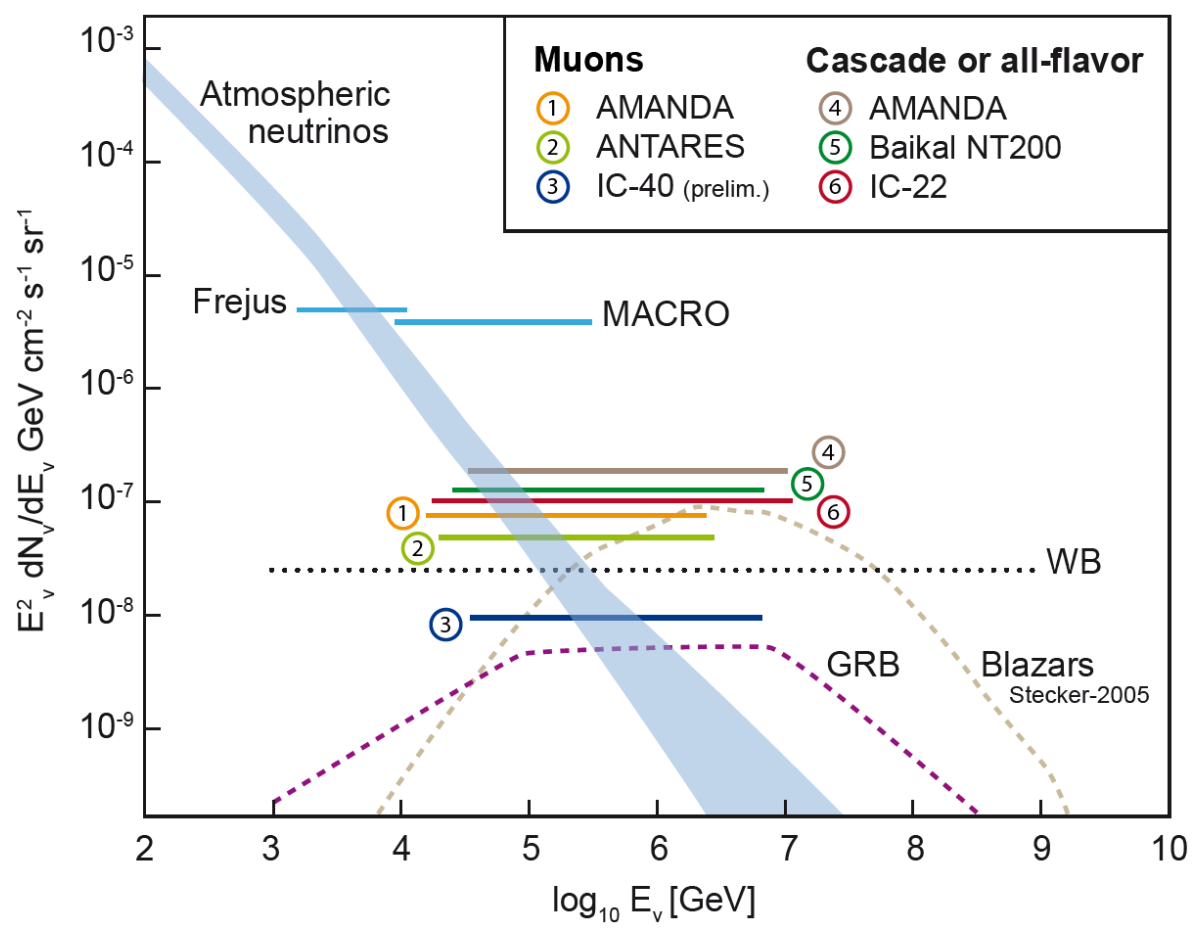

Fig. 22. $90 \%$ C.L. upper limits on the diffuse flux of extraterrestrial neutrinos. The horizontal lines extend over the energy range which would cover $90 \%$ of the detected events from an $E^{-2}$ source (5\% would be below and $5 \%$ above the range). All model predictions have been normalized to one flavor, i.e. all of the all-flavor limits have been divided by 3 . The colored band indicates the measured flux of atmospheric neutrinos (see also Fig. 14), the broadening at higher energies reflects the uncertainties for prompt neutrinos. The limits on muon neutrinos are from 807 days AMANDA 334 days ANTARES and 375 days IceCube-40. Cascade/all flavor limits are from 807 days AMANDA 1038 days Baikal-NT200 257 days IceCube-22. See Katz \& Spiering 2011 for references. Also indicated is the Waxman-Bahcall (WB) bound Waxman \& Bahcall 1999.

If the extraterrestrial signal is not concentrated to individual strong sources but distributed over all the sky, the signal has to be identified in diffuse fluxes. Searches for diffuse fluxes can only use the measured energy as criterion for separating cosmic and atmospheric neutrinos, searching for an excess at high energies. These studies are not restricted to muon neutrino interactions with a muon in the final state. Since the directional information is not of prime importance, one can also study events without a long track but only a particle cascade in the final state. Such events emerge from electron- and tau-neutrino interactions and from all neutral current interactions. Due to neutrino oscillations, two third of the extraterrestrial neutrinos arrive as $\nu_{e}$ or $\nu_{\tau}$. No significant excesses over atmospheric neutrinos or other kinds of background has been observed so far, resulting in upper limits on the diffuse flux of extraterrestrial high energy neutrinos. Figure 22 summarizes the limits obtained in the $\mathrm{TeV}-\mathrm{PeV}$ region. For each experiment and each method only the best limit is shown. Remark- 
ably, from the first limit derived from the underground experiment Frejus (1996) to the 2010 IceCube-40 limit, a factor of 500 improvement has been achieved. Several models such as e.g. the blazar model of Stecker Stecker 2005 shown in the figure can be excluded, and the Waxman-Bahcall bound (see section 8) was eventually passed. A further factor of 10 improvement is expected over the next 2-3 years, using the full IceCube detector and combining muon and cascade information. The expected sensitivity is more than an order of magnitude below the Waxman-Bahcall bound, and prompt atmospheric neutrinos will be detectable for all but the lowest predictions Kowalski 2005.

Five decades after the first conceptual ideas, and three decades after first practical attempts to build high-energy neutrino telescopes, we may be close to a turning point. IceCube, has started data taking in its full cubic-kilometer configuration, provided results equivalent to $1 \mathrm{~km}^{2} \times$ year, but has not yet detected an extraterrestrial neutrino signal.

The strong case for high-energy neutrino astronomy has remained unchanged over time, but the requirements on the necessary sensitivity have tightened continuously. Whereas underground detectors on the kiloton mass scale (or on the $10^{3} \mathrm{~m}^{2}$ muon area scale) seemed sufficient in the sixties, predictions from the seventies and eighties already favored scales of $10^{5}-10^{6} \mathrm{~m}^{2}$. Actually, DUMAND was conceived as a cubic kilometer configuration in 1978. On the other hand, underground detectors like MACRO or Super-Kamiokande were still given a certain potential for high energy neutrino astronomy. Therefore it is no surprise that, in spite of their declared goal of the kilometer scale, also the underwater/ice community did hope for early discoveries with NT200, AMANDA, NESTOR and ANTARES. This hope turned out to be elusive. Neither did the observations of $\mathrm{GeV}$ and $\mathrm{TeV}$ gamma rays in the last two decades support higher flux expectations nor has any of these detectors seen a signal indication with more than $3 \sigma$ significance. Therefore the detection of first extraterrestrial high-energy neutrinos sources lies still ahead. With some optimism, we may expect it within the next few years. Galactic "Pevatrons" such as those observed in gamma rays by the Milagro detector are within reach after a few years of IceCube data taking if the corresponding predictions are correct. Models assigning the most energetic cosmic rays to Gamma Ray Bursts are challenged by recent IceCube data Abbasi 2012 and will be more strongly scrutinized within a couple of years. However, clear detections are all but guaranteed.

\section{Alternative technologies for highest energies - a parallel story}

To detect the feeble fluxes at $100 \mathrm{PeV}-100 \mathrm{EeV}$ (see Fig.1) one needs detectors one to three orders larger than IceCube. The region is dominated by "cosmogenic" neutrinos, a guaranteed source (although with a flux uncertain by 1-2 orders of magnitude) and a possible admixture of neutrinos emerging from jets of AGN. In order to reach such volumes one has to use emissions which propagate with kilometer-scale attenuation and allow a sparse instrumentation. There are three methods to reach such volumes.

- Acoustic detection

- Radio detection

- Detection via air showers

Although the roots of all three methods lay in the seventies and eighties, it was only in the last decade that a broader community got engaged. The actual status of these very dynamic fields is nicely reflected in the series of ARENA Workshops [Nahnhauer 2005 Thompson 2006 Ameli 2008 Latridou 2010]. ARENA stands for Acoustic and Radio EeV Neutrino Detection Activities. Table 4 compiles the corresponding projects, together with the optical instruments underwater and in ice. 


\begin{tabular}{|l|l|l|l|}
\hline Experiment & Location & Technique & Status \\
\hline \hline DUMAND & Hawaii & Water Cherenkov & turned down 1996 \\
\hline NT200+ & Lake Baikal & Water Cherenkov & operating \\
\hline GVD & Lake Baikal & Water Cherenkov & design phase \\
\hline AMANDA & South Pole & Water Cherenkov & terminated 2009 \\
\hline IceCube & South Pole & Water Cherenkov & operating \\
\hline ANTARES & Mediterranean & Water Cherenkov & operating \\
\hline NESTOR & Mediterranean & Water Cherenkov & R\&D for KM3NeT \\
\hline NEMO & Mediterranean & Water Cherenkov & R\&D for KM3NeT \\
\hline KM3NeT & Mediterranean & Water Cherenkov & design phase \\
\hline HIRES & USA & Air shower & terminated 2009 \\
\hline Auger & Argentina & Air shower & operating \\
\hline TA & USA & Air shower & operating \\
\hline JEM-EUSO & Satellite & Air shower & construction \\
\hline ASHRA & Hawaii & air shower & partial operation \\
\hline CRTNT & China & air shower & planned \\
\hline ANITA & Antarctica (balloon) & Radio (ice) & flights continuing \\
\hline RICE & South Pole & Radio (ice) & terminated \\
\hline ARA & South Pole & Radio (ice) & construction stage 1 \\
\hline ARIANNA & Antarctic shelf & Radio (ice) & construction stage 1 \\
\hline SALSA & open & Radio (salt mine) & conceptual phase \\
\hline SAUND & Caribbean Sea & Acoustic & terminated \\
\hline SPATS & South Pole & Acoustic & test array operating \\
\hline AMADEUS & Mediterranean Sea & Acoustic & test array operating \\
\hline ON $\nu$ DE & Mediterranean Sea & Acoustic & test array finished \\
\hline Baikal & Lake Baikal & Acoustic & R\&D \\
\hline GLUE & USA & Radio (moon) & terminated \\
\hline NUMOON & Netherlands & Radio (moon) & operating \\
\hline Kalyzhin & Russia & Radio (moon) & operating \\
\hline LORD & Satellite & Radio (Earth) & planned \\
\hline FORTE & Satellite & terminated \\
\hline
\end{tabular}

Table 4. Instruments and R\&D projects for high energy astrophysical neutrino detection

\subsection{Acoustic detection}

The principle of acoustic particle detection was explained in 3.4. An historical review of acoustic particle detection can be found in Nahnhauer 2010 and a snapshot of the present situation in Nahnhauer 2012. Here, I sketch some of the most important milestones.

Acoustic detection has first been intensively discussed in the context of DUMAND (see section 3.4). Since after 1980 DUMAND focused all forces to the optical method, acoustic detection went dormant until it had a certain revival within the NESTOR project. But also the NESTOR collaboration had its hands full with developing optical equipment, therefore acoustic activities did not really move ahead.

Given the limited resources within the big neutrino underwater projects, in the early nineties Igor Zheleznykh from INR Moscow proposed to use military acoustic arrays in the Black Sea and at the Russian Pacific coast to dig for neutrino signatures. The idea was first realized in the last decade, however not in Russia but by using part of a very large, sparsely instrumented hydrophone array of the US Navy, close to the Bahamas Lehtinen 2002. The array covered an area of $250 \mathrm{~km}^{2}$, could trigger on events above $100 \mathrm{EeV}$ with a tolerable background rate and derived upper limits on the flux of ultra-high energy neutrinos [Kurahashi 2010]. 
Presently, R\&D on acoustic detection is pursued in the Mediterranean Sea close to Sicily (initially running under the name $\mathrm{O} \nu \mathrm{DE}$ ) and to Toulon (project AMADEUS, linked to the ANTARES detector), in Lake Baikal, at the Scottish Coast and at the South Pole (see for references [Nahnhauer 2012] and Katz \& Spiering 2011]). For ice, the signal itself is expected to be higher and ambient noise to be lower than in sea water. A test array, SPATS (South Pole Acoustic Test Setup), has been deployed at the South Pole in order to determine the depth dependence of the speed of sound, Abbasi 2010, the attenuation length of acoustic signals Abbasi 2011b and the ambient noise Abbasi 2012a. The results for the attenuation length is slightly discouraging as the attenuation length turns out to be about $300 \mathrm{~m}$, an order of magnitude smaller than predicted by Buford Price in the nineties Price 1996. However, the noise level seems to be smaller than in the deep sea at a calm sea state.

As a variation of the ice approach, even the use of permafrost as medium has been discussed Nahnhauer 2008.

\subsection{Radio Detection}

In 1983, G. Gusev and I. Zheleznykh proposed the Radio Antartic Muon And Neutrino Detector (RAMAND) with radio antennas "listening" to the Antarctic ice massive Gusev \& Zehelznykh 1983. The proposal was based on an effect predicted in 1962 by Gurgen Askaryan Askaryan 1962. Electromagnetic cascades emit coherent Cherenkov radiation at radio frequencies. It is due to electrons from the material traversed which are swept into the developing shower, which thus acquires an electric net charge. This charge propagates like a relativistic pancake of about $1 \mathrm{~cm}$ thickness and $10 \mathrm{~cm}$ diameter. For wavelengths exceeding the cascade diameter, coherent emission of electromagnetic radiation occurs. The signal amplitude increases with the square of the net charge in the cascade, i.e. it is proportional to $E_{\nu}^{2}$, thus making the method particularly attractive for high-energy cascades. The resulting bipolar pulse is in the radio frequency band and has a width of 1-2 ns. In 2001, the effect was confirmed by measurements at accelerators Saltzberg 2001 Gorham 2007.

In ice, attenuation lengths of several hundred meter to more than a kilometer have been reported for radio signals, depending on the frequency band and the ice temperature. This implies that for energies above hundred $\mathrm{PeV}$ radio detection may become competitive or superior to optical detection (with its attenuation length of the order $100 \mathrm{~m}$ ) Price 1996.

The RAMAND detector of Gusev and Zheleznykh was planned to be deployed at the Soviet Antarctic station Vostok, but with the decay of the Soviet Union the chances for realization faded away. About ten years later, RICE, the Radio Ice Cherenkov Experiment, was installed at the American Amundsen-Scott Station at the South Pole, with 20 receivers and emitters buried at depths between 120 and $300 \mathrm{~m}$. From the non-observation of very large pulses, limits on the diffuse flux of neutrinos with $E>100 \mathrm{PeV}$ and on the flux of relativistic magnetic monopoles have been derived Kravchenko 2006.

Much larger volumes could be observed with the "Antarctic Impulsive Transient Array" (ANITA) Barwick 2006. This is an array of radio antennas which has been flown at a balloon on an Antarctic circumpolar path in 2006 and 2008/09. From $35 \mathrm{~km}$ altitude it searched for radio pulses from neutrino interactions in the thick ice cover and monitored, with a threshold in the range of several $\mathrm{EeV}$, a volume of the order of $10^{6}$ Gigatons. The resulting neutrino flux limits Gorham 2011 are presented in Fig. 23.

Future plans for radio detection in ice foresee large arrays of antennas on the surface of the antarctic ice shelf (project ARIANNA) [Barwick 2007] or in the South 
Polar ice close to surface (project ARA) Allison 2011. Both projects envisage a first stage on the scale of hundred cubic kilometers.

Most exotic is the search for radio emission from extremely-high energy cascades induced by neutrinos or cosmic rays skimming the moon surface. It was first proposed by I. Zheleznykh in 1988 [Zheleznykh 1988] under the name RAMHAND (Radio Astronomical Method of Hadron And Neutrino Detection). After first attempts at the Parks Radio Observatory in the mid nineties, the experiment was firstly realized by the "Goldstone Ultra-high Energy Neutrino Experiment" (GLUE) which used two NASA antennas and reached a maximum sensitivity at several thousand EeV=1000 Gorham 2004. With the same method, the NuMoon experiment at the Westerbork Radio Telescope was searching for extremely energetic neutrinos [Scholten 2008]. Presently, the Kalyzin 65-m radio telescope in Russia is pursuing similar observations. Corresponding activities are also under preparation in the context of the LOFAR experiment Corstanje 2011. An overview of RAMHAND-type activities is given in Dagkesamanksii 2011.

\subsection{Detection via air showers}

At energies above $10^{17} \mathrm{eV}$, large air shower arrays like the Pierre Auger Observatory in Argentina AUGER or the Telescope Array in Utah, USA Sasaki 2003] are searching for horizontal air showers induced by neutrino interactions deep in the atmosphere (showers caused by charged cosmic ray interactions start much higher up in the atmosphere). The optimum sensitivity window for this method is at $1-100 \mathrm{EeV}$. An even better sensitivity might be obtained for tau neutrinos, $\nu_{\tau}$, scratching the Earth and interacting close to the array Fargion 2002 Bertou 2002 Fargion 2004. The charged $\tau$ lepton produced in charged-current interaction can escape the rock around the array (in contrast to electrons) and mostly decays into hadrons (branching ratio ca.65\%) after a short path length (in contrast to muons). If this decay happens in the field of view of the fluorescence telescopes of the Pierre Auger Observatory or the Telescope Array, the decay cascade can be recorded. The limits derived by Auger are included in Fig. 23 .

Space-based observation of extended air showers is an approach to even further increase the target mass for highest-energy cosmic-ray and neutrino detection, at energies beyond $10^{19} \mathrm{EeV}$. This the rationale for the Extreme Universe Space Observatory (EUSO) which has been proposed around the year 2000. With a wide-field camera, EUSO will observe the atmosphere from an orbit at several $100 \mathrm{~km}$ height and register the fluorescence light from extended air showers and, if circumstances allow, also the reflection at Earth surface of the Cherenkov light emitted in shower direction. After several years of technical development and a long phase of uncertainty concerning the space carrier, plans are now to install the device - meanwhile renamed to JEM-EUSO [JEM-EUSO] - on the Japanese Experiment Module (JEM) of the International Space Station, with the launch expected around 2015. JEM-EUSO will observe an atmospheric target volume with a mass of more than one Tera-ton and will thus exceed the Auger sensitivity by two orders of magnitude for energies above some $10^{19} \mathrm{eV}$ (see for the actual status of the mission [Anzalone 2012]).

Figure 23 summarizes the present limits in the PeE-EeV region. One sees that IceCube competes well up to several hundred PeV. At higher energies, ANITA takes over. A realistic chance to measure more than just a handful of cosmogenic neutrinos will stay the privilege of detectors of the 100-1000 cubic kilometer scale. 


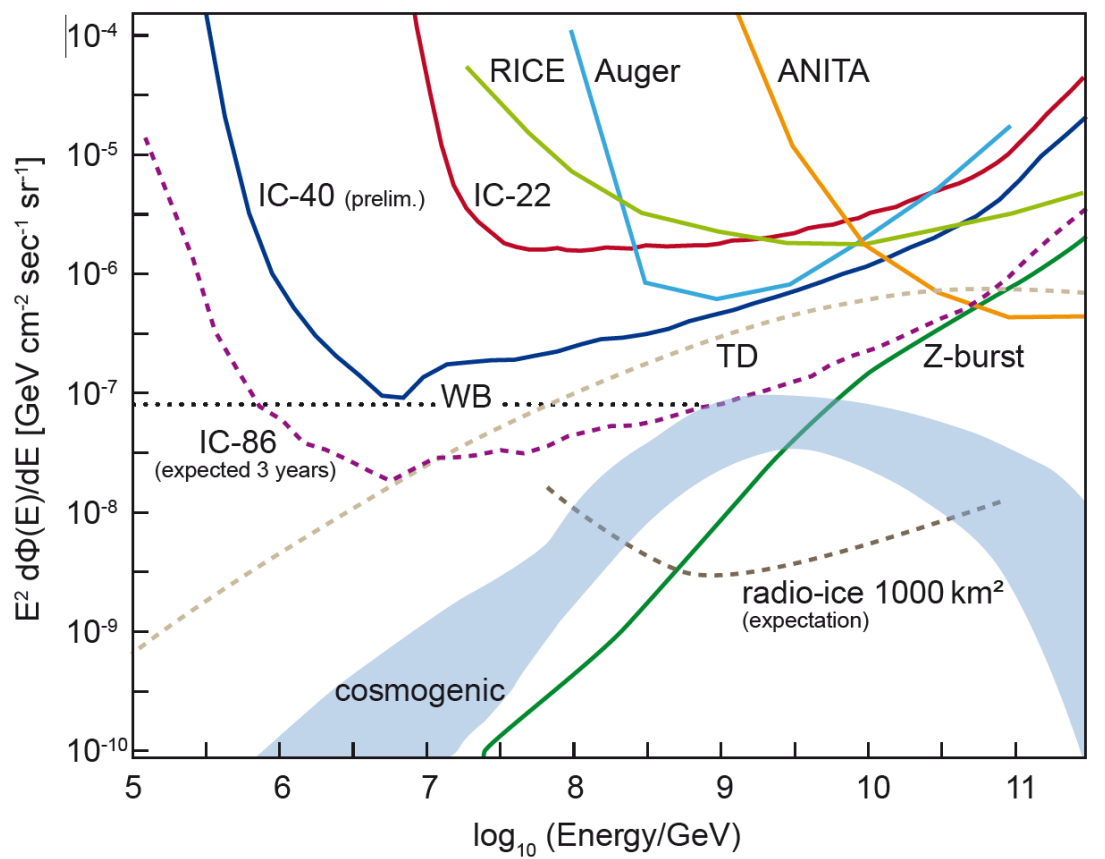

Fig. 23. All-flavour $90 \%$ C.L. differential upper limits on the flux of extraterrestrial neutrinos in the $\mathrm{PeV}-\mathrm{EeV}$ region. Limits are from the under-ice radio array $\mathrm{RICE}$, the air shower detector Auger, the radio balloon experiment ANITA, IceCube-22, and IceCube-40. See for references Katz \& Spiering 2011. Also given are the expectations for 3 years of operation of the full IceCube detector and for a $1000 \mathrm{~km}^{2}$ shallow radio detector at the South Pole. The colored band corresponds to different predictions for cosmogenic neutrinos processes. References for the these scenarios, the Z-burst prediction and the SUSY top-down scenario (TD) can be found in Abbasi 2011c.

\section{What next?}

Whereas the identification of first extraterrestrial neutrinos IceCube has not yet been achieved, projects of similar or greater size on the Northern hemisphere are under preparation. In 2002, an expert committee installed by the International Union of Pure and Applied Physics (IUPAP) concluded HENAP 2002 that "a $\mathrm{km}^{3}$-scale detector in the Northern hemisphere should be built to complement the IceCube detector being constructed at the South Pole". The main physics rationale of this recommendation was that the field of view of a Northern detector includes the central parts of the Galaxy, with several potential neutrino sources. IceCube can see only the outer parts of the galactic plane - with the exception of low energies (DeepCore detecting contained events) and very high energies (where the background from downward moving atmospheric muons becomes small). Following this recommendation, the Mediterranean neutrino telescope groups have formed the KM3NeT collaboration to prepare, construct and operate such a device. KM3NeT has been included in the priority project list of the European Strategy. Forum on Research Infrastructures, ESFRI.

A design study from 2006 to 2009 resulted in a Conceptual Design Report (CDR) Bagley 2008 and a Technical Design Report (TDR) Bagley 2010. At present, the project is in a Preparatory Phase and envisages to install a detector with $6 \mathrm{~km}^{3}$ volume from 2014 on. The total investment cost is estimated to be around 225 MEuro. 
A top view of a possible detector configuration consisting of two blocks, each $3 \mathrm{~km}^{3}$, is sketched in Fig.24.

After initial funding in the Netherlands some years ago, substantial funding for building engineering array(s) has been recently assigned in Italy and in France. At present, the partners are preparing construction of this/these demonstrator(s).

In Russia, the Baikal Collaboration plans the stepwise installation of a kilometerscale array in Lake Baikal, the Gigaton Volume Detector, GVD Aynutdinov 2009. In the years 2008-2010 the basics elements - new optical modules, readout with Flash-ADCs. underwater communication and trigger system - have been tested with stationary prototype strings and resulted in a Conceptual Design Report [GVD 2012]. In April 2012 an engineering array with a first full-scale string and two half-strings have been deployed for a long-term test. Realizing that the originally planned size of half a cubic kilometer is no longer enough, a four times larger array is presently being studied, as sketched bottom-right of Fig.24. Note that due to the shallower depth of Lake Baikal, the height of GVD will be smaller than that of KM3NeT and IceCube.

\section{KM3NeT, Mediterranean Sea, $2 \times 3 \mathrm{~km}^{3}$, sensitive above $\sim 0.5 \mathrm{TeV}$}
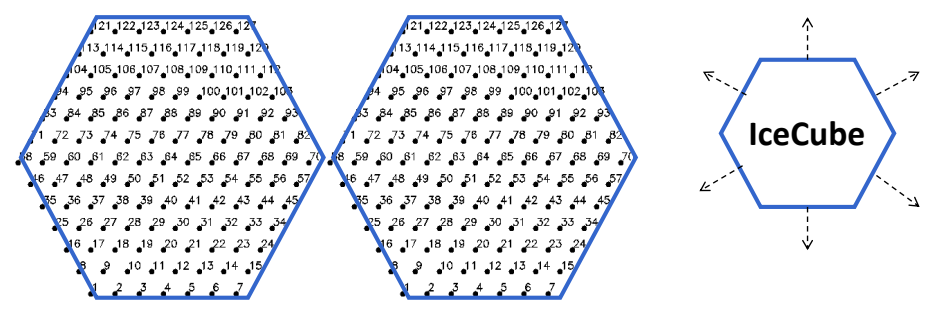

GVD, Lake Baikal, $0.5 \mathrm{~km}^{3}$,
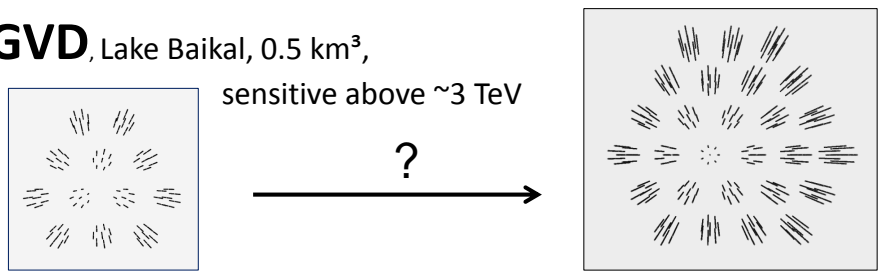

Fig. 24. Top views of planned new detectors at the Northern hemisphere (KM3NeT and GVD). The are compared to the top view of IceCube. Arrows symbolize the possibility of an IceCube extension in case of discovery of extraterrestrial neutrinos.

The realization of these projects depends on several factors. First of all, IceCube results will play a strong role. Secondly, future gamma-ray data must provide stronger indications that the observed gamma-rays are pion-decay counterparts of neutrinos and not only the result of inverse Compton scattering. And last but not least, the considerable funding must be found.

Missing or marginal evidence for sources from IceCube may have various consequences. If one is going to continue the venue of detectors which explore the energy range most characteristic for GRBs and AGNs, one has to envisage an order-ofmagnitude step in sensitivity, i.e. beyond what is presently scheduled by KM3NeT and GVD.

The second option would be an even larger leap in size. It would address energies above $100 \mathrm{PeV}$ with the help of new technologies like radio or acoustic detection and 
envisage 100-1000 cubic kilometers of instrumented volume. This option might still have sensitivity to neutrinos from AGN jets but would also cover well the energy range of neutrinos from cosmic ray interactions with the 3-Kelvin microwave background. In contrast to optical detectors, new-technology detectors are still in the R\&D phase and also have no natural calibration source like atmospheric neutrinos for optical detectors.

The third option would define, at least for the time being, an end to the search for neutrinos from cosmic accelerators. It would focus on optical detection with small spacing optimized to investigate oscillations with accelerator neutrinos (Mediterranean Sea) and atmospheric neutrinos, or, even more pretentious, to study Supernova bursts beyond our own Galaxy or even proton decay.

Taken all together, we may be close to a turning point. We have made a factorof-thousand step in sensitivity compared to a dozen years ago. This is far more than the traditional factor of ten which so often led to the discovery of new phenomena Harwit 1981. For instance, looking across our own field, the prospects for discovery had not been estimated too highly before launching the first X-ray rocket in 1962, or before detecting the Crab Nebula in TeV gamma rays in 1989. History has told another story, as we know today. The same may be the case for high energy neutrino astronomy. The journey is not yet finished!

\section{Acknowledgments}

I want to thank Albrecht Karle, John Learned, Rolf Nahnhauer, Per-Olof Hulth, Alan Watson and Ralf Wischnewski for carefully reading the manuscript and for many extremely helpful comments. I also acknowledge valuable information and comments from Mark Bowen, Grigorij Domogatsky, Zhan-Arys Dzhilkibaev, Stavros Katsanevas, Ulrich Katz, Christopher Wiebusch and Igor Zhelesnykh.

\section{References}

Abbasi, R. et al. (IceCube Coll.) 2009, Search for point sources of high energy neutrinos with final data from AMANDA-II, Phys. Rev. D79: 062001, and arXiv:0809.1646.

Abbasi, R. et al. (IceCube Coll.) 2010, Measurement of sound speed vs. depth in South Pole ice for neutrino astronomy, Astropart. Phys. 33: 277, and arXiv:0909.2629

Abbasi, R. et al. (IceCube Coll.) 2011, Measurement of the atmospheric neutrino energy spectrum from 100 to $400 \mathrm{TeV}$ with IceCube, Phys. Rev. D83: 012001, and arXiv:1010.3980

Abbasi, A. et al. (IceCube Coll.) 2011, IceCube Sensitivity for Low-Energy Neutrinos from Nearby Supernovae, Astron.\& Astrophys. 535: A109, and arXiv:1108.0171.

Abbasi, R. et al. (IceCube Coll.) 2011, Measurement of Acoustic Attenuation in South Pole Ice, Astropart. Phys. 34: 382, and arXiv:1004.1694

Abbasi, R. et al. (IceCube Coll.) 2011, Constraints on the extremely-high energy neutrino flux with the IceCube 2008-2009 data, Phys. Rev. D83: 092003, and arXiv:1103.4250.

Abbasi, A. et al. (IceCube Coll.) 2012, Neutrinos challenge Gamma Ray Burst origin of cosmic rays, Nature 484: 351.

Abbasi, A. et al. (IceCube Coll.) 2012 Background studies for acoustic neutrino detection at the South Pole, Astropart. Phys. 35: 312, and arXiv:1103.1216.

Abraham, J. et al., (Pierre Auger Coll.) 2008, Upper limit on the diffuse flux of UHE tau neutrinos from the Pierre Auger Observatory, Phys. Rev. Lett. 100: 211101, and arXiv:0712.1909

Achar, C. et al 1965., Detection of muons produced by cosmic ray neutrinos deep underground, Phys. Lett. 18: 196. 
Ackermann, M. et al. (AMANDA Coll.) 2006, Optical properties of deep glacial ice at the South Pole, Journ. Geophys. Res. 111: D13203.

Ackermann, M. 2006, Searches for signals from cosmic point-like sources of high energy neutrinos in 5 years of AMANDA-II data, Ph.D. thesis, Humboldt University Berlin. Available from: http://edoc.hu-berlin.de/docviews/abstract.php?lang=ger, id=27726.

Ackermann, M. et al. (AMANDA Coll.) 2006, The IceCube prototype string in AMANDA, Nucl. Instr. Meth. A556: 169, and arXiv:astro-ph/0601397

Ageron, M. et al. (ANTARES Coll.) 2007, Studies of a full-scale mechanical prototype line for the ANTARES neutrino telescope and tests of a prototype instrument for deep-sea acoustic measurements, Nucl. Inst. Meth. A 581: 695.

Ageron, M. et al. (ANTARES Coll.) 2011, ANTARES: The first undersea neutrino telescope, Nucl. Inst. Meth. A656: 11, and arXiv:1104.1607

Aggouras, G. et al. (NESTOR Coll.) 2005, Operation and performance of the NESTOR test detector, Nucl. Inst. Meth. A 552: 420.

Aggouras, G. et al. (NESTOR Coll.) 2005, A measurement of the cosmic-ray muon flux with a module of the NESTOR neutrino telescope, Astropart. Phys. 23: 377.

Aguilar, J.A. et al. (ANTARES Coll.) 2011, A fast algorithm for muon track reconstruction and its application to the ANTARES neutrino telescope, Astropart. Phys. 34: 652.

Ahmad, Q. et al. (SNO Collaboration) 2001, Measurement of the charged current interactions produced by B8 neutrinos at the Sudbury Neutrino Observatory, Phys. Rev. Lett. 87: 071301.

Ahrens, J. et al. (AMANDA Coll.) 2004, Muon track reconstruction and data selection techniques in AMANDA, Nucl. Inst. Meth. A 524: 169, arXiv:astro-ph/0407044.

Ahrens, J. et al. (IceCube Coll.) 2004, Sensitivity of the IceCube detector to astrophysical sources of high energy muon neutrinos, Astropart. Phys. 20: 507, and arXiv:astro$\mathrm{ph} / 0305196$

Aiello, S. et al. (NEMO Coll.) 2010, Measurement of the atmospheric muon flux with the NEMO Phase-1 detector, Astropart. Phys 33: 263, and arXiv:0910.1269

Allison, P. et al. 2011, Design and Initial Performance of the Askaryan Radio Array Prototype EeV Neutrino Detector at the South Pole, submitted to Astropart. Phys., arXiv:1105.2854.

Alvarez-Muniz, J. and Halzen; F. 2002 Possible high-energy neutrinos from the cosmic accelerator RXJ 1713.7-3946, Astrophys. J. 576: 233, and arXiv:astro-ph/0205408.

Ameli, F. (ed.) 2009, Proc. 3rd Int. ARENA Workshop, Univ. Rome, 2008, Nucl. Instr. Meth. Suppl. A604.

Amram, P. etal. (ANTARES Coll.) 2000, Background light in potential sites for the ANTARES undersea neutrino telescope, Astropart. Phys. 13: 127.

Amram, P. et al. (ANTARES Coll.) 2003, Sedimentation and fouling of optical surfaces at the ANTARES site, arXiv:astro-ph/0206454.

Andres, E. et al. (AMANDA Coll.) 1999, The AMANDA Neutrino Telescope: Principle of Operation and First Results, Astropart. Phys. 13: 1, and arXiv:astro-ph/9906203

Andres, E. et al. (Amanda Coll.) 2001, Results from the AMANDA high-energy neutrino detector, Nucl. Phys. Proc. Suppl. 91 : 423, and arXiv:astro-ph/0009242.

ANTARES homepage: http://antares.in2p3.fr

Antonioli, P. et al. 2004, SNEWS: The supernova early warning system, New J. Phys. 6: 114, and arXiv:astro-ph/040621.

Anzalone, A, et al. 2012, The JEM-EUSO Mission: Status and Prospects, Contributions of the JEM-EUSO Collaboration to the 32nd ICRC, Beijing 2011, arXiv:1204.5065.

Araki, T. et al. 2005 (Kamland Collaboration) Experimental investigation of geologically produced antineutrinos with KamLAND, Nature 436: 499.

Askaryan, G. 1957, Hydrodynamic radiation from the tracks of ionizing particles in stable liquids, Sov. J. Atom. Energy 3: 921.

Askaryan, G. 1962, Excess negative charge of an electron-photon shower and its coherent radio emission, Sov. Phys. JETP 14: 441.

Askebjer, P. et al. (AMANDA Coll.) 1995, Optical properties of the South Pole ice at depths between $0.8 \mathrm{~km}$ and $1 \mathrm{~km}$, Science 267: 1147 . 
Aslanides, E. et al. (ANTARES Coll.) 1999, A deep sea telescope for high-energy neutrinos, arXiv:astro-ph/9907432.

AUGER, Pierre Auger Observatory homepage: http://www.auger.org

Avronin, A. et al. (Baikal Coll.) 2009, Search for high-energy Neutrinos in the Baikal Neutrino Experiment, Astron. Lett. 35: 650.

Aynutdinov, V. et al. (Baikal Coll.) 2006, Search for a diffuse flux of high-energy extraterrestrial neutrinos with the NT200 neutrino telescope, Astropart. Phys. 25: 140, and arXiv:astro-ph/0508675.

Aynutdinov, V. et al. (Baikal Coll.) 2006, The BAIKAL neutrino experiment: From NT200 to NT200+, Nucl. Inst. Meth. A 567: 433, and arXiv:astro-ph/0609743

Aynutdinov, V. etal. (Baikal Coll.) 2009, The prototype string for the km3-scale Baikal neutrino telescope, Nucl. Inst. Meth. A 602:227, and and arXiv:0811.1110

Babson, E. et al. (DUMAND Coll.) 1990, Cosmic ray muons in the deep ocean, Phys. Rev. D 42: 3613.

Bagduev, R. et al. (Baikal Coll.) 1999, The optical module of the Baikal deep underwater neutrino telescope, Nucl. Inst. Meth. A 420: 138, and arXiv:astro-ph/9903347.

Bagley, P. et al. (KM3NeT Coll.) 2008, Conceptual design report, ISBN 978-90-6488-031-5, available from: www.km3net.org.

Bagley, P. et al. (KM3NeT Coll.) 2010, Technical design report, ISBN 978-90-6488-033-9, available from: www.km3net.org.

Bahcall, J. 1994, in: Solar Neutrinos - the first thirty years, Addison-Wesley Publ. Comp., Reading.

BAIKAL, Baikal homepage: http://baikalweb.jinr.ru

Boliev, M. et al. 1981, Limitations on parameters of neutrino oscillations according to data of the Baksan underground telescope (in Russian) Yad. Fiz. 34: 1418.

Balkanov, R. et al. (Baikal Coll.) 1997, Reconstruction of atmospheric neutrinos with the Baikal neutrino telescope NT-96, Astropart. Phys. 12: 75, and arXiv:astro-ph/9705244.

Barwick, S. et al. (ANITA Coll.) 2006, Constraints on cosmic neutrino fluxes from the ANITA experiment, Phys. Rev. Lett. 96: 171101, and arXiv:astro-ph/0512265

Barwick, S. 2007, ARIANNA: A new concept for UHE neutrino detection, Journ. Phys. Conf. Ser. 60: 278, and arXiv:astro-ph/0610631.

Bellini, G. et al. 2010 (Borexino Collaboration), Observation of Geo-Neutrinos, Phys. Lett.B 687: 299.

Belolaptikov, I. et al., The experimental limits on Q-ball flux with the Baikal deep underwater array Gyrlyanda, arXiv:astro-ph/9802223.

Belotti, E. and Laveder, M. 1993, High energy neutrino detectors, Proc. 5th Int. Workshop on Neutrino Telescopes(ed. M.Baldo-Ceolin), Venice: 275.

Berezinsky, V. and Smirnov, A. 1975, Cosmic Neutrinos of Ultra-High Energies and Detection Possibility, Astrophys. Space Science 32: 461.

Berezinsky, V. and Zatsepin, G. 1970, Cosmic neutrinos of superhigh energies, Yad. Fiz. 11: 200.

Berezinsky, V. and Priludsky, O., High Energy Neutrinos from Supernova Explosions and Davis' Experiment, Sov. Astron. Lett. 3: 79.

Berezinsky, V. 1990, High energy neutrino astronomy, Proc. Int. Workshop on Neutrino Telescopes (ed. M.Baldo-Ceolin), Venice: 125.

Bergeson, H. etal. 1967, Evidence for a new production process for $10^{12} \mathrm{eV}$ muons, Phys. Rev. Lett. 19: 1487.

Bergeson, H., Cassiday, G. and Hendricks, M. 1973, Phys. Rev. Lett. 31: 66.

Bertou, X. et al. 2002, Tau Neutrinos in the Auger Observatory: a new Window on UHECR Sources, Astropart. Phys. 17:183.

Bezrukov, L.B. et al. (Baikal Coll.) 1984, Progress report on Lake Baikal neutrino experiment: Site studies and stationary string, Proc. XI. Conf. on Neutrino Physics and Astrophysics, Nordkirchen, Germany: 550.

Bezrukov, L.B. etal. 1987, Properties and test results of a photon detector based on the combination of electro-optical preamplifier and a small photomultiplier, Proc. 2nd Int. Symp. Underground Physics-87, Baksan Valley, USSR: 230. 
Blondeau, F. for the ANTARES Coll. 1998, The ANTARES demonstrator: Towards a highenergy undersea neutrino telescope, Prog. Part. Nucl. Phys. 40: 413.

Bobisut, F. 1991, NET: a Neutrino Telescope, Proc. 3nd Int. Workshop on Neutrino Telescope (ed. M. Baldo-Ceolin), Venice: 387.

Bogatyrev, V. 1971, On the possibility of constructing large detectors for neutrino astronomy, Soviet J. of Nucl. Phys. 13: 187.

Bosetti, P. et al. (DUMAND Coll.) 1988, DUMAND II: Proposal to construct a deep-ocean laboratory for the study of high energy neutrino astrophysics and particle physics, Tech. Rep. HDC-2-88, Hawaii DUMAND Center, University of Hawaii.

Bosetti, P. 1991, JULIA, Proc. Trends in Astroparticle Physcis (ed. Bosetti, P.), Aachen 1991: 188

Bradner, H. (ed.) 1977, Proc. Workshop on acoustic detection of atmospheric neutrinos (DUMAND 77)

Capone, A. et al. (NEMO Coll.) 2009, Recent results and perspectives of the NEMO project, Nucl. Inst. Meth. A 602: 47.

Corstanje, A. et al. 2011, LOFAR: Detecting Cosmic Rays with a Radio Telescope, Contribution to 32nd ICRC, Beijing, 2011, arXiv:1109.5805.

Costantini, M. and Vissani, F. 2005, Neutrinos from supernovas and supernova remnants, arXiv:astro-ph/0508152.

Cowan, C. et al. 1956, Detection of the Free Neutrino: A Confirmation, Science 103: 124.

Cowsik, R. et al. 1963, Flux of atmospheric neutrinos of different types at sea level and cosmic ray neutrino experiments, in Proc. Int. Cosmic Ray Conf. 1963, 6: 211.

Crouch, M. et al. 1978, Cosmic-ray muon fluxes deep underground. Intensity vs. depth and the neutrino-induced component, Phys. Rev. D18: 2239.

Dagkesamanksii, R., Matveev, V. and Zheleznykh, I. 2011 Prospects of radio detection of extremely high energy neutrinos bombarding the moon, Nucl. Instr. Meth. A626: S44.

Davis Jr., R et al. 1968 A search for neutrinos from the Sun, Phys. Rev. Lett. 20: 1205.

Deneyko, A.O. et al. 1991, The tests of a prototype of an autonomous module of deep underwater neutrino telescope during October-December of 1989, Proc. 3rd Int. Workshop on Neutrino Telescopes, Venice: 407.

De Rujula, D. and Glashow, S. 1984 Nuclearites - a novel form of cosmic radiation, Nature 312: 734 .

Dirac, P. 1931, Quantised singularities in the electromagnetic field, Proc. Royal Soc. of London A133: 60 .

Distefano, C. 2007 Detection potential to point-like neutrino sources with the NEMO-km3 telescope, Astrophys. Space Sci. 309: 415, and arXiv:astro-ph/068514.

Domogatsky, G. and Zatsepin, G. 1965, On the experimental possibilities of the observation of neutrinos from collapsing stars, Proc. 9th Int. Conf. on Cosmic Rays, London: 1030.

Domogatsky, G. etal. 1986, Present status of Baikal deep underwater experiment, Proc. XII. Conf. on Neutrino Physics and Astrophysics, Sendai, Japan: 737.

Eberl, T. for the ANTARES Coll. 2011, Status and first results of the ANTARES neutrino telescope, Prog. Part. Nucl. Phys. 66: 457.

Fargion, D. 2002, Discovering ultra high energy neutrinos by horizontal and upward tau air-showers: Evidences in terrestrial gamma flashes?, Astrophys. Journ. 570: 909, and arXiv:astro-ph/0002453.

Fargion, D., De Sanctis Lucentini, P. and De Santis, M. 2004, Tau air showers from Earth, Astrophys. Journ. 613: 1285, and arXiv:hep-ph/0305128

Feinstein, F. for the ANTARES Coll. 1999, The ANTARES demonstrator towards an undersea neutrino telescope, Nucl. Phys. Proc. Suppl. 70: 445.

Fermi, E. 1934, Versuch einer Theorie der $\beta$-Strahlen, Zeitschr. f. Physik 88: 161.

Fukuda, Y. et al. (Super-Kamiokande Coll.) 1998 Evidence for oscillation of atmospheric neutrinos Phys. Rev. Lett. 81: 1562.

Gaisser, T. 1990, Prospects for neutrino astronomy, Proc. 2nd Workshop on Neutrino Telescopes (ed. M. Baldo-Ceolin), Venice: 397.

Gorham, P. et al. 2004, Experimental limit on the cosmic diffuse ultra-high energy neutrino flux, Phys. Rev. Lett. 93: 0041101, and arXiv:astro-ph/0310232. 
Gorham, P. et al. 2007, Observations of the Askaryan effect in ice, Phys. Rev. Lett. 99: 171101, and arXiv:hep-ex/0611008

Gorham, P. et al. 2011, Observational constraints on the ultra-high energy cosmic neutrino flux from the second flight of the ANITA experiment, Erratum to Phys. Rev. D82: 022004, arXiv:1011.5004

Greisen, K. 1960, Cosmic Ray Showers, Ann. Rev. Nucl. Part. Sci. 10: 63.

Grieder, P. 1994 DUMAND: Facts, Figures and Initial Operation, Proc. XVI Int. Conf. on Neutrino Physics and Astrophysics, Eilat 1994.

GVD, Gigaton Volume Detector, Design Report 2012 (in Russian) at http://baikalweb.jinr.ru

Gusev, G. and Zhelesnykh, I. 1983, On the possibility of detection of neutrinos and muons on the basis of radio radiation of cascades in natural dielectric media. JETP Letters 38: 611.

Halzen, F. and Learned, J.G. 1988, High Energy Neutrino Detection in Deep Polar Ice, in Proc. 5th Int. Symp. on Very High-Energy Cosmic-Ray Interactions, Lodz, Poland.

Halzen, F. and Learned, J. 1993, High energy neutrino astronomy: towards a 1 km3 detector, Proc. 5th Int. Workshop on Neutrino Telescopes (ed. Milla Baldo-Ceolin), Venice: 483.

Halzen, F. 1995, Ice fishing for neutrinos Available from: http://icecube.berkeley.edu/amanda/ice-fishing.html

Halzen, F. 1998, Antarctic Dreams, Available from: http://www.exploratorium.edu/origins/antarctica/tools/dreams1.html

Halzen, F. and Hooper, D. 2005, High energy neutrinos from the TeV blazar 1ES 1959+650, Astropart. Phys. 23: 537, and arXiv:astro-ph/0502449

Harwit, M. 1981, Cosmic Discovery, Basic Books Inc., New York.

Heisenberg, W. 1936, Zur Theorie der Schauerbildung in der Höhenstrahlung, Zeitschr. f. Physik 101, 533.

The High Energy Neutrino Astrophysics Panel, E. Fernandez et al., High energy neutrino observatories, available from: www.lngs.infn.it/lngs/infn/contents/docs/pdf/panagic/henap2002.pdf.

Hess, V. 1912, Über die Bedeutung der durchdringenden Strahlung bei sieben Freiluftballonfahrten, Phys. Zeitschr. 12: 998.

IceCube homepage: http://www.icecube.wisc.edu/.

IceCube: a Kilometer-Scale Neutrino Observatory. A Proposal to the National Science Foundation. The U.S. institutions of the IceCube Collaboration, Nov.1, 1999.

JEM-EUSO homepage: http://jemeuso.riken.jp/en/index.html

Johannson, S. 1991, Detection of High Energy Neutrinos, Proc. XXIIth ICRC, Dublin: 552.

Kampert, K.-H. and Watson, A. 2012, Extensive Air Showers and Ultra High-Energy Cosmic Rays: A Historical Review, to be published in "Cosmic Rays, Gamma Rays and Neutrinos: a Survey of 100 Years of Research" (ed. C. Spiering), Europ. Phys. Journ. H, 2012.

Kappes, A. et al. 2007, Potential neutrino signals from galactic gamma-ray sources, Astrophys. Journ. 656: 870, and arXiv:astro-ph/0607286

Karaevsky, S. et al. 1993, Sea Acoustic Detection of Cosmic Objects (SADCO), Proc. 23rd ICRC, Calgary, 4: 550 .

Katz, U. and Spiering, C. 2012 High-energy neutrino astrophysics: Status and perspectives, to be published in Progress in Particle and Nuclear Physics, arXiv: 1111-0507.

Kistler, M. and Beacom, J. 2006, Guaranteed and prospective galactic TeV neutrino sources, Phys. Rev. D74: 063007, and arXiv:astro-ph/0607082.

Kotzer, P. (ed.) 1976, DUMAND-75, Proc. 1975 Summer DUMAND Study, Western Washington State College, Bellingham, WA.

Krishnaswamy, M. et al. 1971, The Kolar Gold Fields Neutrino Experiment. I. The Interactions of Cosmic Ray Neutrinos, Proc. Roy. Soc. London 323: 489.

Kowalski, M. 2005, Measuring diffuse neutrino fluxes with IceCube, Journ. Cosm. Astropart. Phys. 0505: 010, and arXiv:astro-ph/0505506.

Kropp, W. and Crouch, M. 1991, Some Reminiscences of the CWI Atmospheric Neutrino Experiment, in Neutrinos and Other Matter (Selected Works of Frederick Reines), World Scientific: 226 . 
Kurahashi, N., Vandenbroucke, J. and Gratta, G. 2010 Phys. Rev. D82: 073006.

Kravchenko, I. et al. 2006, RICE limits on the diffuse ultra-high energy neutrino flux, Phys. Rev. D73: 082002, and arXiv:astro-ph/0601148.

Latridou, P. (ed.) 2010, Proc. fourth Int. ARENA Workshop, 2010 Nantes, Nucl. Inst. Meth. $62(2012)$

Learned, J. (ed.) 1979, DUMAND-1979. Proc. of Khabarovsk and Lake Baikal Summer Workshops.

Learned, J. 1979a, Acoustic radiation by charged atomic particles in liquids: An analysis, Phys. Rev. D19: 3239. (REPLACE?)

Learned, J. 1990, Future of high energy neutrino astronomy, Proc. 2nd Int. Workshop on Neutrino Telescope (ed. M. Baldo-Ceolin), Venice: 103

Learned, J. and Pakvasa S. 1995, Detecting $\nu_{\tau}$ Oscillations at PeV Energies, Astropart. Phys. 3: 276 , and arXiv:hep-ph/9408296

Lehtinen, N. et al. 2002, Sensitivity of an underwater acoustic array to ultra-high energy neutrinos, Astropart. Phys. 17: 279, and arXiv:astro-ph/010433.

Lorenz, E. and Wagner, R. 2012, Very high-energy gamma ray astronomy: a 23-year success story, to be published in "Cosmic Rays, Gamma Rays and Neutrinos: a Survey of 100 Years of Research" (ed. C. Spiering), Europ. Phys. Journ. H, 2012.

Lowder, D. et al. 1991, Observation of muons using the polar ice cap as a Cherenkov detector, Nature 353: 331.

Mannheim, K., Protheroe, R. and Rachen, J. 2001, On the cosmic ray bound for models of extragalactic neutrino production, Phys. Rev. D63: 023003, and astro-ph/9812398

Markov, M.A. 1960, On high energy neutrino physics, in: Proc. 10th ICHEP, Rochester: 578.

Markov, M. and Zheleznykh, I. 1960, On high energy neutrino physics in cosmic rays, in the collection "On high energy neutrino physics", Dubna 1960, p. 17.

Markov, M. and Zheleznykh, I. 1961, On high energy neutrino physics in cosmic rays, Nucl. Phys. 27: 385.

Markov, M. 1993, Rasmyshlyaja o fisikakh, o fisike, o mire ("contemplating on physics, physicists and the world", in Russian), Nauka 1993: 76.

McDonald, A. et al. 2004, Astrophysical neutrino telescopes, Rev. Sci. Instrum. 75: 293, and arXiv:astro-ph/0311343

Morse, R. 1993, The AMANDA Astronomy Project, Proc. 5th Int. Workshop on Neutrino Telescope (ed. M. Baldo-Ceolin), Venice: 309.

Morlino, G., Blasi, P. and Amato, E. 2009, Gamma rays and neutrinos from supernova remnant RXJ 1713.7.3946, Astropart. Phys. 31: 376, and arXiv:0903.4565

Nahnhauer, R. and Boeser, S. (eds.) 2006, Proc. Int. ARENA Workshop, Zeuthen 2005, World Sci. Publ. Co. PTE LTD, Singapore.

Nahnhauer, R., Rostovtsev, A. and Tosi, D. 2008, Permafrost - an alternative target material for ultra high energy neutrino detection?, Nucl. Instr. Meth. A29: 29, and arXiv:0707.3757.

Nahnhauer, R. 2010, Acoustic particle detection - from early ideas to future benefits, Nucl. Instrum. Meth. A662: 20, and arXiv:1010.3082.

Nahnhauer, R. 2012 Acoustic detetction of ultra-high energy neutrinos: a snapshot, to appear in Nucl. Instrum. Meth., and arXiv:1201.0908

Narisimham, V. 2004, Perspectives of Experimental Neutrino Physics in India, Proc. Indian Natn. Science Acad., 70A: 11.

NEMO homepage: http://nemoweb.lns.infn.it

NESTOR homepage: http://www.nestor.noa.gr/

Nishikawa, K. 1992, Titel Proc.4th Int. Workshop on Neutrino Telescopes (ed.M. BaldoCeolin) Venice: 337.

Osborne, J., Said, S. and Wolfendale A. 1965, The energy spectra of cosmic ray neutrinos at ground level in the range 1-1000 GeV, Proc. Phys. Soc. 86: 93.

Pauli, W. 1930, "Liebe radioaktive Damen und Herren", Letter printed in Physics Today 31N9 (1978): 72.

Pistilli, P. 1988, SINGAO: a very large telescope for neutrino and gamma astronomy and cosmic ray studies, Proc. Int. Workshop on Neutrino Telescope (ed. M. Baldo-Ceolin): 316. 
Price, B. 1996, Comparison of optical, radio, and acoustical detectors for ultrahigh-energy neutrinos, Astropart. Phys. 5: 43.

Reimer, A., Böttcher, M. and Postnikov, S. 2005, Neutrino emission in the hadronic synchrotron mirror model: the "orphan" TeV flare from 1ES 1959+650, Astrophys. J. 630: 186, and arXiv:astro-ph/0505233.

Reines, F. 1960, Neutrino Interactions, Ann. Rev. Nucl. Part. Sci. 10: 1.

Reines, F. etal. 1965., Evidence for high-energy cosmic-ray neutrino interactions, Phys. Rev. Lett. 15: 9.

Reines, F. 1981, Closing summary $\nu-81$, in: V. Peterson (ed.), Proc. 30th Int. Conf. on Neutrino Physics and Astrophysics, Vol. 2: 496.

Resvanis, L. et al. (NESTOR Coll.) 1994, NESTOR: A neutrino particle astrophysics underwater laboratory for the Mediterranean, Nucl. Phys. Proc. Suppl. 35: 294.

Roberts, A. (ed.) 1976, DUMAND-76, Proc. 1976 Summer DUMAND Workshop, University of Hawaii, Honolulu, HI.

Roberts, A. and Wilkins, G. (eds.) 1978, DUMAND-78, Proc. 1978 Summer DUMAND Study.

Roberts, A. 1992, The birth of high-energy neutrino astronomy: A personal history of the DUMAND project, Rev. Mod. Phys. 64: 259.

Rubakov, V. 1981, Superheavy monopoles and proton decay, JETP Lett. 33: 644.

Saltzberg, D. et al. 2001, Observation of the Askaryan effect: Coherent microwave Cherenkov emission from charge asymmetry in high energy particle cascades, Phys. Rev. Lett.: 2802, and arXiv:hep-ex/0011001.

Sasaki, M., Asaoha, Y. and Jobashi, M. 2003, Detecting very high energy neutrinos by the Telescope Array, Astropart. Phys. 19: 37, and arXiv:astro-ph/0204167

Scholten, O. et al. 2008, Improved flux limits for neutrinos with energies above $10^{22}$ ev from observations with the Westerbork Synthesis Radio Telescope, Phys. Rev. Lett. 103: 191301, and arXiv:0910.4745

Sobel, H. 1988, The GRANDE facility for the study of astrophysical sources and high-energy particle interactions, Proc. Int. Workshop on Neutrino Telescope (ed.M. Baldo-Ceolin), Venice: 233.

Sokalski, I. and Spiering, C., eds. (Baikal Coll.) 1992, The Baikal Neutrino Telescope NT-200, Tech. Rep. Baikal-92-03, DESY/INR.

Spiering, C. 2011, Neutrino Detectors under Water and Ice, Landolt-Börnstein, New Series I, 21B2, 6.2. Springer Verlag 2011.

Stecker, F.W. 2005, A note on high energy neutrinos from AGN cores, Phys. Rev. D 72: 107301, and arXiv:astro-ph/0510537

Stenger, V. et al. (eds.) 1992 Proc. Workshop High Energy Neutrino Astrophysics, World Scientific 1992.

Stenger, V. and Learned, J. 1992, AGN Event Rates in DUMAND II, Proc. Workshop High Energy Neutrino Astrophysics, World Scientific 1992: 288.

Svoboda, R. et al., 1987 (IMB Coll.), An upper limit on the flux of extraterrestrial neutrinos, Astrophys. J. 315: 420.

Taiuti, M. etal. (NEMO Coll.) 2011, The NEMO project: A status report, Nucl. Inst. Meth. A 626: S25.

Thompson,L. and Danahe, S. (eds.) 2007, Proc. 2nd Int. ARENA Workshop, Univ. Northumbria, 2006, Journ. of Phys. Conf. Series 81.

Uberall, H. and Cowan, C. 1965, Proposal to detect cosmic-ray neutrinos through the Cerenkov light of produced muons, Proc. CERN Conf. on Experimental Neutrino Physics (ed. C. Franzinetti), CERN 65-32: 496.

van Aller, G. et al. 1986, A "smart" 35-cm Diameter Photomultiplier, Helvetica Physica Acta 59: 1119.

Volkova, L. and Zatsepin, G., Energetic spectra of muon and electron neutrinos in the atmosphere, Isv. AN USSR, physics series 29/9: 1749

Waxman, E. and Bahcall, J. 1999, High-energy neutrinos from astrophysical sources: An upper bound, Phys. Rev. D 59: 023002, and arXiv:hep-ph/9807282 
Weekes, T.C. et al. 1989, Observation of TeV gamma rays from the Crab nebula using the atmospheric Cerenkov imaging technique, Astrophys. J. 342: 379.

Wiebusch, C. 1995, The detection of faint light in deep underwater neutrino telescopes, PhD thesis, report PITHA 95/37 (Univ. Aachen).

Wischnewski, R. et.al. 1993, The Lake Baikal Telescope NT-36 - a first deep underwater multi-string array, Proc. 3rd Int. NESTOR Workshop, Pylos, Greece: 213.

Zatsepin, G. and Kuzmin, V. 1961 Neutrino production in the atmosphere, Soviet J. Exp. Theor. Physics (JETP) 41: 385.

Zheleznykh, I. 1988, Prospects for large scale detectors of super high-energy neutrinos $\left(10^{15}\right.$ to $10^{20} \mathrm{eV}$ ), Proc. 13rd Int. Conf. Neutrino Physics and Astrophysics: 528.

Zheleznykh, I. 2006, Early years of high-energy neutrino physics in cosmic rays and neutrino astronomy, Int. J. Mod.Pंhys. A21S1: 1. 